\title{
Review and Principles of PPP-RTK Methods
}

\author{
P. J. G. Teunissen - A. Khodabandeh
}

Received: date / Accepted: date

\begin{abstract}
PPP-RTK is integer ambiguity resolution enabled precise point positioning. In this contribution we present the principles of PPP-RTK, together with a review of different mechanizations that have been proposed in the literature. By application of $\mathcal{S}$-system theory, the estimable parameters of the different methods are identified and compared. Their interpretation is essential for gaining a proper insight into PPP-RTK in general, and into the role of the PPP-RTK corrections in particular.
\end{abstract}

We show that PPP-RTK is a relative-technique for which the 'single-receiver user' integer ambiguities are in fact double differenced ambiguities. We determine the transformational links between the different methods and their PPP-RTK corrections, thereby showing how different PPP-RTK methods can be mixed between network and users. We also present and discuss four different estimators of the PPP-RTK corrections. It is shown how they apply to the different PPP-RTK models, as well as why some of the proposed estimation methods cannot be accepted as PPP-RTK proper. We determine analytical expressions for the variance matrices of the ambiguity-fixed and ambiguity-float PPPRTK corrections. This gives important insight into their precision, as well as allows us to discuss which parts of

P. J. G. Teunissen

GNSS Research Centre, Department of Spatial Sciences, Curtin University of Technology, Perth, Australia; Department of Geoscience and Remote Sensing, Delft University of Technology, Delft, The Netherlands

E-mail: p.teunissen@curtin.edu.au

A. Khodabandeh

GNSS Research Centre, Department of Spatial Sciences, Curtin

University of Technology, Perth, Australia

E-mail: amir.khodabandeh@curtin.edu.au the PPP-RTK correction variance matrix are essential for the user and which are not.

Keywords PPP-RTK, Integer Ambiguity Resolution, Ambiguity Dilution of Precision (ADOP), $\mathcal{S}$-bases, Common Clock (CC) model, Distinct Clock (DC) model, Integer Recovery Clock (IRC) model, Decoupled Satellite Clock (DSC) model, Fractional Cycle Bias (FCB) model, Geometry-Free (GF) model, Geometrybased (GB) model

\section{Introduction}

PPP-RTK is integer ambiguity resolution enabled precise point positioning (PPP) (Wubbena et al, 2005; Mervart et al, 2008; Teunissen et al, 2010). With PPP, precise satellite orbits and clocks are provided to enable single-receiver users to compute their receiver-positions with a high, decimeter or centimeter, accuracy (Zumberge et al, 1997; Kouba and Heroux, 2001; Bisnath and Gao, 2008). PPP-RTK extends the PPP-concept by providing single-receiver users, next to the orbits and clocks, also information about the satellite phase biases. This information, when properly provided, enables recovery of the integerness of the user-ambiguities, thus enabling single-receiver ambiguity resolution thereby reducing the convergence times as compared to that of PPP. The goal of this contribution is to present the principles of PPP-RTK, together with a review of the different mechanizations that have been proposed in the literature.

In recent years, several PPP-RTK methods have been proposed and formulated, see e.g., Wubbena et al (2005); Laurichesse and Mercier (2007); Mervart et al (2008); Collins (2008); Ge et al (2008); Bertiger et al (2010); Teunissen et al (2010); Geng et al (2012); Lannes 
and Prieur (2013). These methods differ in the models used, in the corrections applied and/or in the estimation methods employed. Although some comparative studies between some of these different PPP-RTK methods already exist, these studies have not been sufficiently conclusive. The method comparisons of Geng et al (2010) and Shi and Gao (2013), for instance, do not identify some of the important differences that exist between the methods. Instead they state that the methods studied are theoretically equivalent and will provide equivalent results. This finding is echoed in the publications of, for instance, Bisnath and Collins (2012, p. 378), Shi (2012, p. 89), Li et al (2013a, p. 4), and Zhang and $\mathrm{Li}(2013$, p. 580). We will show, however, that there are differences between the methods, even up to the point that some cannot be accepted as proper PPPRTK methods.

It is the purpose of this contribution to present a framework describing the intricate elements of PPPRTK, which then are used to identify and describe the differences and similarities of the different methods. We make a distinction between the model formulation used and the estimation method employed. We discuss both the network-component and the user-component. Furthermore, by a careful application of $\mathcal{S}$-system theory (Teunissen, 1985), we are in the position to give a clear description of the estimable parameters that are involved in the various different methods. The interpretation of these estimable parameters is essential for gaining a proper insight into PPP-RTK in general, and into the role of the PPP-RTK corrections in particular.

This contribution is organized as follows. After having discussed the basic idea of single-receiver integer ambiguity resolution, three different PPP-RTK models are presented in section 2, two based on different versions of the common-clock (CC) model and one based on the distinct-clock (DC) model. In section 3, we discuss some of the popular ionosphere-free PPP-RTK models. They are the Integer Recovery Clock (IRC) model (Laurichesse and Mercier, 2007; Laurichesse et al, 2009; Laurichesse, 2011; Loyer et al, 2012), the Decoupled Satellite Clock (DSC) model (Collins, 2008; Collins et al, 2008), and the Uncalibrated Phase Delay/Fractional Cycle Bias (UPD/FCB) model (Ge et al, 2008; Geng, 2011). They are compared mutually as well as with the methods of section 2 .

In section 4 we discuss the role of the PPP-RTK corrections in establishing the link between the userparameters and the network-parameters. The corrections are designed to realize integer ambiguities in the user-equations, thus enabling user integer ambiguity resolution. We show that PPP-RTK is a relative-positioning method and that these 'single-receiver user' inte- ger ambiguities are straightforward classical double differenced (DD) ambiguities and thus not undifferenced ambiguities as is sometimes stated. We also show how the different PPP-RTK corrections are related. This has the important practical implication that it shows how the different PPP-RTK methods can be mixed between network and users.

Section 5 deals with the estimation of the PPPRTK corrections. Four different estimators of the corrections are discussed. They are the float and fixed estimators under the geometry-free (GF) model and the float and fixed estimators under the geometry-based (GB) model (Teunissen, 1997a). An analytical formulation of their precision is presented, which is then used to compare the performance of the different estimators. It is shown how each of these estimators apply to the different PPP-RTK models. The variance matrices of the individual PPP-RTK corrections are also used to determine the variance matrix of the complete usercorrections. Here we identify which parts of the PPPRTK correction variance matrix are essential for the user and which are not. Finally, in Section 6, our estimation results are compared to the estimation method as described for FCB. The characteristics of the differences between the PPP-RTK estimators is demonstrated and conclusions on their suitability are drawn.

We make use of the following notation: The expectation and dispersion operators are denoted as $E($. and $\mathrm{D}($.$) , respectively. In case distributional results are$ given, the observables are assumed to follow a (multivariate) normal distribution. The identity matrix of order $n$ is denoted as $I_{n} .\lfloor x\rceil$ denotes the nearest integer to $x$. If $x$ is a vector, then $\lfloor x\rceil$ is the vector that follows from component-wise integer rounding. The betweensatellite single-differenced (SD) combinations are symbolized through $(.)^{p s}=(.)^{s}-(.)^{p}$, with $s$ and $p$ being the rover and pivot satellites, respectively. A similar notation is used for between-receiver differences, $(.)_{q u}=(.)_{u}-(.)_{q}$, with $u$ and $q$ being the rover and pivot receiver, respectively. The squared norm of vector, with respect to positive-definite matrix $Q$, is symbolized by $\|.\|_{Q}^{2}=(.)^{T} Q^{-1}($.$) .$

\section{PPP-RTK: From Network to User}

\subsection{Single-Receiver Integer Ambiguity Resolution}

The idea of single-receiver integer ambiguity resolution (IAR) forms the basis of PPP-RTK. The basic idea of single-receiver IAR is best described by starting with the single-receiver user observation equations. Here and in the following we will be working with between-satellite single-differenced observation equations, instead of with 
the undifferenced observation equations. This simplifies our presentation, but does not affect the generality and results of our analyses.

Consider the user's antenna $u$ tracking dual-frequency GNSS data that are transmitted by a rover satellite $s$ and a chosen pivot satellite $p$. The corresponding between-satellite single-difference (SD) observation equations read then (Hofmann-Wellenhof et al, 2008)

$$
\begin{aligned}
& \Delta \phi_{u, j}^{p s}=g^{p s T} \Delta x_{u}-\mu_{j} \iota_{u}^{p s}-d t^{p s}+\lambda_{j}\left(z_{u, j}^{p s}-\delta_{, j}^{p s}\right) \\
& \Delta p_{u, j}^{p s}=g^{p s T} \Delta x_{u}+\mu_{j} \iota_{u}^{p s}-d t^{p s}-d_{, j}^{p s}
\end{aligned}
$$

where $\Delta \phi_{u, j}^{p s}$ and $\Delta p_{u, j}^{p s}$ denote the SD 'observed-minuscomputed' phase and code observables on the frequency band $f_{j}(j=1,2)$, respectively. Here and in the following, the precise orbital corrections are assumed included in the 'observed-minus-computed' observables. The $\nu$-vector $\Delta x_{u}$ contains the user's position increments and/or the zenith tropospheric delay (ZTD). Parameter $\nu$ can take the values $\nu=3$ (position-only model), $\nu=1$ (ZTD-only model) or $\nu=4$ (positionplus-ZTD model). Thus the $\nu$-vector $g^{p s}$ contains the $\mathrm{SD}$ receiver-satellite unit vector and/or the SD tropospheric mapping function. The (first-order) SD slant ionospheric delay, experienced on the first frequency, is denoted by $\iota_{u}^{p s}$. Thus the frequency-dependent coefficients are defined as the ratio $\mu_{j}=\left(f_{1}^{2} / f_{j}^{2}\right)$. The SD integer ambiguity $z_{u, j}^{p s} \in \mathbb{Z}$ and the SD satellite phase bias $\delta_{, j}^{p s}$, both expressed in cycles, are linked to the phase observables through the wavelength $\lambda_{j}$. The SD satellite clocks are denoted by $d t^{p s}$, while the SD satellite code biases are denoted by $d_{, j}^{p s}$. Apart from $z_{u, j}^{p s}$ and $\delta_{, j}^{p s}$, the rest of the quantities are all expressed in units of range. We assume that $m$ satellites are tracked and thus $p, s=1, \ldots, m$, with $p \neq s$.

If we make use of the more compact dual-frequency vector notation $\Delta \phi_{u}^{p s}=\left[\Delta \phi_{u, 1}^{p s}, \Delta \phi_{u, 2}^{p s}\right]^{T}, \Delta p_{u}^{p s}=\left[\Delta p_{u, 1}^{p s},-\right.$ $\left.\Delta p_{u, 2}^{p s}\right]^{T}, \mu=\left[\mu_{1}, \mu_{2}\right]^{T}, z_{u}^{p s}=\left[z_{u, 1}^{p s}, z_{u, 2}^{p s}\right]^{T}, \delta^{p s}=\left[\delta_{, 1}^{p s}, \delta_{, 2}^{p s}\right]^{T}$, and $d^{p s}=\left[d_{, 1}^{p s}, d_{, 2}^{p s}\right]^{T}$, we may write (1) as

$$
\begin{aligned}
& \Delta \phi_{u}^{p s}=e \Delta \rho_{u}^{p s}-\mu \iota_{u}^{p s}-e d t^{p s}+\Lambda\left(z_{u}^{p s}-\delta^{p s}\right) \\
& \Delta p_{u}^{p s}=e \Delta \rho_{u}^{p s}+\mu \iota_{u}^{p s}-e d t^{p s}-d^{p s} \\
& \text { where } e=[1,1]^{T}, \Lambda=\operatorname{diag}\left(\lambda_{1}, \lambda_{2}\right), \text { and } \\
& \Delta \rho_{u}^{p s}=g^{p s T} \Delta x_{u}
\end{aligned}
$$

The user observation equations (2) do not contain enough information to solve for an integer ambiguity resolved user position. This would become possible though, were information about the satellite clocks and satellite biases be given. Using such externally provided information to correct the observations as

$$
\begin{aligned}
& \Delta \phi_{u}^{\prime p s}=\Delta \phi_{u}^{p s}+e d t^{p s}+\Lambda \delta^{p s} \\
& \Delta p_{u}^{\prime p s}=\Delta p_{u}^{p s}+e d t^{p s}+d^{p s}
\end{aligned}
$$

results in user-equations that take the form

$$
\begin{aligned}
& \Delta \phi_{u}^{\prime p s}=e \Delta \rho_{u}^{p s}-\mu \iota_{u}^{p s}+\Lambda z_{u}^{p s} \\
& \Delta p_{u}^{\prime p s}=e \Delta \rho_{u}^{p s}+\mu \iota_{u}^{p s}
\end{aligned}
$$

This system is now in a form that can be used to solve for the integer ambiguity resolved user parameters $\Delta x_{u}$ and $\iota_{u}$. Hence, with externally provided corrections $d t^{p s}, \delta^{p s}$, and $d^{p s}$, the user system of observation equations (5) can be solved as a mixed-integer system of equations, thereby profiting from the integerness of $z_{u}^{p s} \in \mathbb{Z}^{2}$ (Teunissen et al, 2010). This is the basic idea of single-receiver, IAR-enabled, positioning.

The question is now whether the above needed parameters $d t^{p s}, \delta^{p s}, d^{p s}$ can be determined as such. As we will see, the answer is no. Does this mean that the above basic idea is flawed. The answer is again, fortunately, no. In the following we will namely show that although a GNSS-network is not capable of providing the 'absolute' parameters $d t^{p s}, \delta^{p s}, d^{p s}$, it is capable of providing estimable parameters, that - when applied as corrections - achieve the same goal, namely of enabling the construction of a user system of observation equations that is in mixed-integer form.

We apply $\mathcal{S}$-system theory (Baarda, 1973; Teunissen, 1985) to solve for the rank-deficient system of observation equations and to allow for a proper interpretation of the estimable parameters. Different sets of estimable parameters, each with their own interpretation, exist. Each such set is defined by the chosen $\mathcal{S}$-basis. By means of the $\mathcal{S}$-transformation, the relation between the original 'absolute' parameters and the estimable parameters is established. Examples of the theory's applicability to GNSS can be found in de Jonge (1998) and Odijk (2002), while examples for PPP-RTK can be found in Teunissen et al (2010); Zhang et al (2011); Lannes and Teunissen (2011) and Odijk et al (2012).

\subsection{Common Clock (CC-1) Model}

The externally provided satellite clock and satellite biases will be determined by a GNSS-network. We now show how this network information enables the construction of a user system of observation equations that has the same structure as (5).

\subsubsection{Network model}

If we replace the user-index $u$ in $(2)$ by $r=1, \ldots, n$, the resulting system may be considered the dual-frequency $n$-receiver network system of observation equations. Although in the network case, some or all of the entries of $\Delta x_{r}, r=1, \ldots, n$, may be known, we consider the 
general case that they are unknown. This difference is of no consequence for the conclusions of our analysis. Furthermore, it is also sufficient for the purpose of this contribution to assume the network to be such that $g_{r}^{s} \approx g^{s}, r=1, \ldots, n$. This assumption allows the inclusion of small to regional networks in our discussion as well.

With $m$ satellites tracked, the network system of observation equations will have a rank defect of $\nu+$ $4(m-1)$. Of this defect, $\nu$ is due to the linear dependence that exist between the coefficients of $\Delta x_{r}$ and $d t^{p s}$, while $4(m-1)$ is due to the linear dependency among the ionospheric delays, clocks, biases and ambiguities. This latter defect is best demonstrated if we use the geometry-free/ionosphere-free decomposition

$d^{p s}=[\mu, e]\left[\begin{array}{l}d_{G F}^{p s} \\ d_{I F}^{p s}\end{array}\right]$, with $[\mu, e]^{-1}=\left[\begin{array}{l}\mu_{G F}^{T} \\ \mu_{I F}^{T}\end{array}\right]$

to rewrite (2), with $u$ replaced by $r=1, \ldots, n$, as

$\Delta \phi_{r}^{p s}=e \Delta \rho_{r}^{p s}-\mu \tilde{\iota}_{r}^{p s}-e d \bar{t}^{p s}+\Lambda\left(z_{r}^{p s}-\delta^{p s}-M d^{p s}\right)$

$\Delta p_{r}^{p s}=e \Delta \rho_{r}^{p s}+\mu \tilde{\iota}_{r}^{p s}-e d \bar{t}^{p s}$

where $\tilde{\iota}_{r}^{p s}=\iota_{r}^{p s}-d_{G F}^{p s}, d \bar{t}^{p s}=d t^{p s}+d_{I F}^{p s}$ and $M=$ $\Lambda^{-1}\left(\mu \mu_{G F}^{T}-e \mu_{I F}^{T}\right)$. This shows, since only $2(m-1)$ parameters in the combination $z_{u}^{p s}-\delta^{p s}-M d^{p s}$ are estimable, that the additional defect is indeed $4(m-1)$.

There are many different ways of eliminating the rank defect of the above network system of equations (Teunissen, 1985). The $\nu$-defect between $\Delta x_{r}$ and $d t^{p s}$ can be eliminated by fixing the parameters of one of the network stations, say $\Delta x_{1}$. Likewise, the $4(m-1)$-defect between ambiguities, code biases and phase biases, can be eliminated by fixing two out of the three type of parameters, say the ambiguities of network station 1 and the code biases. With this choice the $\mathcal{S}$-basis is thus given as

$x_{C C-1}^{\mathcal{S}}=\left[\Delta x_{1}^{T}, d^{p s T}, z_{1}^{p s T}\right]^{T}$

The full rank network system of observation equations $(r=1, \ldots, n)$ follows then as,

$$
\begin{aligned}
& \Delta \phi_{r}^{p s}=e \Delta \tilde{\rho}_{r}^{p s}-\mu \tilde{\iota}_{r}^{p s}-e d \tilde{t}^{p s}+\Lambda\left(\tilde{z}_{r}^{p s}-\tilde{\delta}^{p s}\right) \\
& \Delta p_{r}^{p s}=e \Delta \tilde{\rho}_{r}^{p s}+\mu \tilde{\iota}_{r}^{p s}-e d \tilde{t}^{p s}
\end{aligned}
$$

in which the estimable parameters, denoted using the tilde ${ }^{\sim}$ symbol, have the following interpretation,

$$
\begin{aligned}
\Delta \tilde{x}_{r} & =\Delta x_{r}-\Delta x_{1}, r \neq 1 \\
\tilde{\iota}_{r}^{p s} & =\iota_{r}^{p s}-\mu_{G F}^{T} d^{p s} \\
d \tilde{t}^{p s} & =d t^{p s}+\mu_{I F}^{T} d^{p s}-\Delta \rho_{1}^{p s} \\
\tilde{\delta}^{p s} & =\delta^{p s}+M d^{p s}-z_{1}^{p s} \\
\tilde{z}_{r}^{p s} & =z_{r}^{p s}-z_{1}^{p s}, r \neq 1
\end{aligned}
$$

with $\Delta \tilde{\rho}_{r}^{p s}$ in (9) denoting $g^{p s T} \Delta \tilde{x}_{r}$. The network vector of estimable parameters of the above model is thus given for a SD satellite pair $p s$ and a network station $r$ as

$x_{C C-1}=\left[\Delta \tilde{x}_{r}^{T}, \tilde{\iota}_{r}^{p s}, d \tilde{t}^{p s}, \tilde{\delta}^{p s T}, \tilde{z}_{r}^{p s T}\right]^{T}$

The system (9) is referred to as the common clock (CC1) model, since the phase and code equations have the satellite clock parameter $d \tilde{t}^{p s}$ in common. This model was used in Zhang et al (2011); Odijk et al (2012). Another common clock model (CC-2), based on a different $\mathcal{S}$-basis, will be presented in section 2.4 .

It is important to recognize that the estimable parameters are not the original parameters, but instead functions of them. The between-satellite estimable slant ionospheric delay $\tau_{r}^{p s}$, for instance, is a biased version of the actual between-satellite slant ionospheric delay $\iota_{r}^{p s}$, and the between-satellite estimable integer ambiguity $\tilde{z}_{r}^{p s}$ is actually a double-differenced integer ambiguity, namely $z_{r}^{p s}$ biased by $-z_{1}^{p s}$. It is furthermore important to recognize that the interpretation of these estimable parameters depends on the chosen $\mathcal{S}$-basis, i.e. it will change when a different $\mathcal{S}$-basis is chosen (Teunissen, 1985). In the next sections, some such cases are described.

\subsubsection{User model}

With appropriate corrections, one should be able to formulate the user-model in mixed-integer form. In case of the above CC-model, the parameters for the corrections are given as

$x_{C C-1}^{c o r r}=\left[d \tilde{t}^{p s}, \tilde{\delta}^{p s T}\right]^{T}$

These are not the original 'absolute' satellite clock and satellite biases, $d t^{p s}$ and $\delta^{p s}$, but instead the satellite clock-like and satellite bias-like terms, $d \tilde{t}^{p s}$ and $\tilde{\delta}^{p s}$, of (10). Although they are not the original parameters, they still do the job in ensuring that the user can work with integer ambiguities. With the CC-corrected observations

$$
\begin{aligned}
& \Delta \tilde{\phi}_{u}^{p s}=\Delta \phi_{u}^{p s}+e d \tilde{t}^{p s}+\Lambda \tilde{\delta}^{p s} \\
& \Delta \tilde{p}_{u}^{p s}=\Delta p_{u}^{p s}+e d \tilde{t}^{p s}
\end{aligned}
$$

the user equations take namely the form

$$
\begin{aligned}
\Delta \tilde{\phi}_{u}^{p s} & =e \Delta \tilde{\rho}_{u}^{p s}-\mu \tilde{\iota}_{u}^{p s}+\Lambda \tilde{z}_{u}^{p s} \\
\Delta \tilde{p}_{u}^{p s} & =e \Delta \tilde{\rho}_{u}^{p s}+\mu \tilde{\iota}_{u}^{p s}
\end{aligned}
$$

Note that the structure of these equations is indeed identical to that of (5) and that the user-ambiguities are indeed integer $\tilde{z}_{u}^{p s} \in \mathbb{Z}^{2}$. The interpretation of the parameters in (14) is however different from those of (5). 
Through the network-based satellite clock corrections $d \tilde{t}^{p s}$, for instance, the positional link between network and user is established, thus giving, instead of $\Delta x_{u}$ in (5), the estimable user parameter vector $\Delta \tilde{x}_{u}$ in (14).

A similar link between network and user is established for the ambiguities. Note namely that the integer 'user' ambiguity $\tilde{z}_{u}^{p s}$ is in fact a double-differenced ambiguity $\tilde{z}_{u}^{p s}=z_{u}^{p s}-z_{1}^{p s}$. In other words, for the ambiguities, the linkage with the network is established through the network-based phase-bias correction $\tilde{\delta}^{p s}$, thus enabling the construction of double-differenced integer ambiguities at the user-side. This shows that one must be very careful by calling the ambiguity resolution of the user-ambiguities, the fixing of undifferenced or single-differenced integer ambiguities, see e.g., Laurichesse et al (2009, p. 135) or Mervart et al (2013, p. 1177). The resolution of these 'user' ambiguities is namely again a resolution of double-differenced ambiguities.

\subsection{Distinct Clocks (DC) Model}

Instead of working with the CC-model, one can also work with distinct clocks (DC) models. Using $\mathcal{S}$-system theory, de Jonge (1998) introduced various $\mathcal{S}$-systems for the undifferenced GNSS observation equations. Since his choices give rise to models with common and/or different clocks for different observable types, de Jonge refers to his models as the distinct clocks (DC) models, see de Jonge (1998, Chapter 4). Distinct clock models were also used in Odijk (2002) and in Teunissen et al (2010) for PPP-RTK. The DC-model of de Jonge that we consider in the present contribution, uses a common clock for the code observables and two different clocks for the two phase observables.

The DC-model uses the same $\mathcal{S}$-basis (8), but a different parametrization. The DC $\mathcal{S}$-basis is thus also given as

$x_{D C}^{\mathcal{S}}=\left[\Delta x_{1}^{T}, d^{p s T}, z_{1}^{p s T}\right]^{T}$

The CC-to-DC reparametrization is rather simple as it only involves replacing the phase bias by a lumped version of the common clock and phase bias,

$\left[\begin{array}{l}d \tilde{t}^{p s} \\ \delta \tilde{t}^{p s}\end{array}\right]=\left[\begin{array}{ll}1 & 0 \\ e & \Lambda\end{array}\right]\left[\begin{array}{c}d \tilde{t}^{p s} \\ \tilde{\delta}^{p s}\end{array}\right]$

Thus instead of the CC-parametrization $d \tilde{t}^{p s}, \tilde{\delta}^{p s}$, now the DC-parametrization $d \tilde{t}^{p s}, \delta \tilde{t}^{p s}$ is used. Its full rank network system of observation equations reads therefore

$$
\begin{aligned}
& \Delta \phi_{r}^{p s}=e \Delta \tilde{\rho}_{r}^{p s}-\mu \tilde{\iota}_{r}^{p s}-\delta \tilde{t}^{p s}+\Lambda \tilde{z}_{r}^{p s} \\
& \Delta p_{r}^{p s}=e \Delta \tilde{\rho}_{r}^{p s}+\mu \tilde{\iota}_{r}^{p s}-e d \tilde{t}^{p s}
\end{aligned}
$$

For a SD satellite pair $p s$ and a network station $r$, the network vector of estimable parameters is then given as:

$x_{D C}=\left[\Delta \tilde{x}_{r}^{T}, \tilde{\iota}_{r}^{p s}, d \tilde{t}^{p s}, \delta \tilde{t}^{p s T}, \tilde{z}_{r}^{p s T}\right]^{T}$

The PPP-RTK corrections of the DC-model are given as

$x_{D C}^{c o r r}=\left[d \tilde{t}^{p s}, \delta \tilde{t}^{p s T}\right]^{T}$

Once these corrections are applied at the user side, the observation equations of the user will again be given by (14). Following the terminology of Laurichesse and Mercier (2007), the correction vector $\delta \tilde{t}^{p s}$ could be called an integer recovery phase clock vector as it results in integer double-differenced ambiguities at the user side.

\subsection{Common Clock (CC-2) Model}

Instead of using the ambiguities of a reference station as part of the S-basis (cf. 8), one may also choose the phase biases themselves. This will result in real-valued ambiguities that can be used to form the PPP-RTK user-corrections.

\subsubsection{Network model}

In case the phase biases $\delta^{p s}$ replace the ambiguities $z_{1}^{p s}$ of (8), the $\mathcal{S}$-basis becomes

$$
x_{C C-2}^{\mathcal{S}}=\left[\Delta x_{1}^{T}, d^{p s T}, \delta^{p s T}\right]^{T}
$$

The full rank network system of observation equations $(r=1, \ldots, n)$ follows then as,

$\Delta \phi_{r}^{p s}=e \Delta \tilde{\rho}_{r}^{p s}-\mu \tilde{\iota}_{r}^{p s}-e d \tilde{t}^{p s}+\Lambda \tilde{a}_{r}^{p s}$

$\Delta p_{r}^{p s}=e \Delta \tilde{\rho}_{r}^{p s}+\mu \tilde{\iota}_{r}^{p s}-e d \tilde{t}^{p s}$

with the estimable parameters,

$$
\begin{aligned}
\Delta \tilde{x}_{r} & =\Delta x_{r}-\Delta x_{1} \\
\tilde{\iota}_{r}^{p s} & =\iota_{r}^{p s}-\mu_{G F}^{T} d^{p s} \\
d \tilde{t}^{p s} & =d t^{p s}+\mu_{I F}^{T} d^{p s}-\Delta \rho_{1}^{p s} \\
\tilde{a}_{r}^{p s} & =z_{r}^{p s}-\delta^{p s}-M d^{p s}
\end{aligned}
$$

When we compare (10) with (22), we note that $\tilde{\delta}^{p s}$ and $\tilde{z}_{r}^{p s}$ of $(10)$, and $\tilde{a}_{r}^{p s}$ of $(22)$, are related as

$\tilde{a}_{r}^{p s}=\tilde{z}_{r}^{p s}-\tilde{\delta}^{p s}$

We thus see that as a consequence of the change of $\mathcal{S}$-basis from $z_{1}^{p s}$ to $\delta^{p s}$ (cf. 8, 20), the $n-1$ integer ambiguity vectors $\tilde{z}_{r}^{p s}$ (recall that $\tilde{z}_{r=1}^{p s}=0$ ), together with the phase bias vector $\tilde{\delta}^{p s}$, get replaced by the $n$ real-valued ambiguity vectors $\tilde{a}_{r}^{p s}$ of (22). The network vector of estimable parameters of this model, for a SD satellite pair $p s$ and a network station $r$, is thus given as

$x_{C C-2}=\left[\Delta \tilde{x}_{r}^{T}, \tilde{\iota}_{r}^{p s}, d \tilde{t}^{p s}, \tilde{a}_{r}^{p s T}\right]^{T}$ 


\subsubsection{User model}

It is now the real-valued ambiguity vector $\tilde{a}_{q}^{p s}$, that, for some $q \in\{1, \ldots, n\}$, can take over the role of the phase bias $\tilde{\delta}^{p s}$ in forming the corrections for the user,

$x_{C C-2}^{c o r r}=\left[d \tilde{t}^{p s}, \tilde{a}_{q}^{p s T}\right]^{T}$

Note that $\tilde{a}_{q}^{p s}=-\tilde{\delta}^{p s}$ for $q=1$ (cf. 23) and $\tilde{a}_{q}^{p s}=$ $\tilde{z}_{q}^{p s}-\tilde{\delta}^{p s}$ for $q \neq 1$. Thus, apart from the sign, the realvalued ambiguity $\tilde{a}_{q}^{p s}$ is either equal to the phase bias or a nonzero integer-shifted version of it. Hence, with the real-valued ambiguity vector $\tilde{a}_{q}^{p s}$ one should indeed be able to recover ambiguity-integerness at the user side. This is verified when such correction is applied to the user observations.

With the user observations corrected as

$\Delta \tilde{\tilde{\phi}}_{u}^{p s}=\Delta \phi_{u}^{p s}+e d \tilde{t}^{p s}-\Lambda \tilde{a}_{q}^{p s}$

$\Delta \tilde{p}_{u}^{p s}=\Delta p_{u}^{p s}+e d \tilde{t}^{p s}$

the user equations take the form

$\Delta \tilde{\tilde{\phi}}_{u}^{p s}=e \Delta \tilde{\rho}_{u}^{p s}-\mu \tilde{\iota}_{u}^{p s}+\Lambda \tilde{a}_{q u}^{p s}$

$\Delta \tilde{p}_{u}^{p s}=e \Delta \tilde{\rho}_{u}^{p s}+\mu \tilde{\iota}_{u}^{p s}$

with integer $\tilde{a}_{q u}^{p s}=z_{u}^{p s}-z_{q}^{p s}$. Thus for $q=1$, the parametrization of (27) is identical to that of (14), while for $q \neq 1$, the ambiguities are an integer shifted version of those of (14). Hence, the ambiguity solution of any network station $q=1, \ldots, n$ can be taken to form an admissible user-correction.

\section{Ionosphere-Free PPP-AR Models}

In this section we discuss some further models proposed in the literature. We discuss the Integer Recovery Clock (IRC) model (Laurichesse and Mercier, 2007; Laurichesse et al, 2009; Loyer et al, 2012), the Decoupled Satellite Clock (DSC) model (Collins, 2008; Collins et al, 2008), and the Uncalibrated Phase Delay/Fractional Cycle Bias (UPD/FCB) model (Ge et al, 2008; Geng and Bock, 2013). Since the IRC- and DSCmodel are the same, they are discussed under one heading.

\subsection{The IRC/DSC Model}

The IRC-model has been introduced in Laurichesse and Mercier (2007) and the DSC-model in Collins (2008). The IRC/DSC-model works with ionosphere-free combinations. Thus instead of working with the four equations of (7), the ionosphere is eliminated first, thereby reducing the four equations to three instead. Although this elimination step is not essential, we include it in the below derivation to better appreciate the choice of parametrization. We show that the IRC/DSC-model uses the same $\mathcal{S}$-basis as the CC-model (9) or DC-model (17), but a different parametrization.

\subsubsection{Network model}

With the wide-lane, narrow-lane and ionosphere-free combinations defined as

$\mu_{W L}=\frac{1}{\sqrt{\mu_{2}}-\sqrt{\mu_{1}}}\left[\sqrt{\mu_{2}},-\sqrt{\mu_{1}}\right]^{T}$,

$\mu_{N L}=\frac{1}{\sqrt{\mu_{2}}+\sqrt{\mu_{1}}}\left[\sqrt{\mu_{2}}, \sqrt{\mu_{1}}\right]^{T}$,

$\mu_{I F}=\frac{1}{\mu_{12}}\left[\mu_{2},-\mu_{1}\right]^{T}$,

we have the properties

$\left[\begin{array}{ccr}\mu_{I F} & 0 & \mu_{W L} \\ 0 & \mu_{I F} & -\mu_{N L}\end{array}\right] \perp\left[\begin{array}{r}-\mu \\ \mu\end{array}\right],\left[\begin{array}{r}\mu_{W L} \\ -\mu_{N L}\end{array}\right] \perp\left[\begin{array}{l}e \\ e\end{array}\right]$

with $\mu_{W L}^{T} e=1$. The notation $v \perp w$ means $v^{T} w=0$. Hence, if the full-rank $4 \times 3$ matrix of (29) is used to form the three ionosphere-free observation combinations $\phi_{r, I F}^{p s}=\mu_{I F}^{T} \phi_{r}^{p s}, p_{r, I F}^{p s}=\mu_{I F}^{T} p_{r}^{p s}$, and $\phi_{r, W N}^{p s}=$ $\mu_{W L}^{T} \phi_{r}^{p s}-\mu_{N L}^{T} p_{r}^{p s}$, their observation equations follow from (9) as

$\Delta \phi_{r, I F}^{p s}=\Delta \tilde{\rho}_{r}^{p s}-d \tilde{t}^{p s}+\mu_{I F}^{T} \Lambda\left(\tilde{z}_{r}^{p s}-\tilde{\delta}^{p s}\right)$

$\Delta p_{r, I F}^{p s}=\Delta \tilde{\rho}_{r}^{p s}-d \tilde{t}^{p s}$

$\Delta \phi_{r, W N}^{p s}=$

$+\mu_{W L}^{T} \Lambda\left(\tilde{z}_{r}^{p s}-\tilde{\delta}^{p s}\right)$

These equations are referred to as 'ionosphere-free' as the ionospheric delay parameters are eliminated from them. These equations are however still in the form of the original CC-parametrization. With the IRC/DSCparametrization

$\left[\begin{array}{c}d \tilde{t}^{p s} \\ \delta \tilde{t}_{I F}^{p s} \\ \tilde{\delta}_{W}^{p s}\end{array}\right]=\left[\begin{array}{cc}1 & 0 \\ 1 & \mu_{I F}^{T} \Lambda \\ 0 & \frac{1}{\lambda_{W}} \mu_{W L}^{T} \Lambda\end{array}\right]\left[\begin{array}{c}d \tilde{t}^{p s} \\ \tilde{\delta}^{p s}\end{array}\right]$,

the wide-lane transformation

$\left[\begin{array}{c}\tilde{z}_{r, 1}^{p s} \\ \tilde{z}_{r, W}^{p s}\end{array}\right]=Z_{W} \tilde{z}_{r}^{p s}$, with $Z_{W}=\left[\begin{array}{rr}1 & 0 \\ 1 & -1\end{array}\right]$,

thereby recognizing that

$\left[\begin{array}{c}\mu_{I F}^{T} \Lambda Z_{W} \\ \mu_{W L}^{T} \Lambda Z_{W}\end{array}\right]=\left[\begin{array}{cc}\lambda_{N} & \frac{\lambda_{2}}{\mu_{12}} \\ 0 & \lambda_{W}\end{array}\right]$

we obtain

$\Delta \phi_{r, I F}^{p s}=\Delta \tilde{\rho}_{r}^{p s}-\delta \tilde{t}_{I F}^{p s}+\lambda_{N} \tilde{z}_{r, 1}^{p s}+\frac{\lambda_{2}}{\mu_{12}} \tilde{z}_{r, W}^{p s}$

$\Delta p_{r, I F}^{p s}=\Delta \tilde{\rho}_{r}^{p s}-d \tilde{t}^{p s}$

$\Delta \phi_{r, W N}^{p s}=\lambda_{W}\left(\tilde{z}_{r, W}^{p s}-\tilde{\delta}_{W}^{p s}\right)$ 
with the narrow-lane and wide-lane wavelengths given as $\lambda_{N}=\left(\lambda_{1} \lambda_{2}\right) /\left(\lambda_{2}+\lambda_{1}\right)$ and $\lambda_{W}=\left(\lambda_{1} \lambda_{2}\right) /\left(\lambda_{2}-\lambda_{1}\right)$. Since $\tilde{z}_{r, 1}^{p s}$ has the narrow-lane wavelength $\lambda_{N}$ as coefficient, it is sometimes referred to as the narrow-lane ambiguity (Beutler et al, 2007). Formally this is incorrect, since $\tilde{z}_{r, 1}^{p s}$ is an $L_{1}$ ambiguity, while the narrowlane ambiguity is defined as the sum of an $L_{1}$ and $L_{2}$ ambiguity (Wubbena, 1989; Allison, 1991).

The estimable IRC/DSC-parameters of (34) have the following interpretation,

$$
\begin{aligned}
& \Delta \tilde{x}_{r}=\Delta x_{r}-\Delta x_{1} \\
& d \tilde{t}^{p s}=d t^{p s}+\mu_{I F}^{T} d^{p s}-\Delta \rho_{1}^{p s} \\
& \delta \tilde{t}_{I F}^{p s}=d t^{p s}+\mu_{I F}^{T} \Lambda\left(\delta^{p s}-z_{1}^{p s}\right)-\Delta \rho_{1}^{p s} \\
& \tilde{\delta}_{W}^{p s}=(0,1) Z_{W}\left(\delta^{p s}-z_{1}^{p s}\right)-\frac{1}{\lambda_{W}} \mu_{N L}^{T} d^{p s} \\
& \left.\begin{array}{l}
\tilde{z}_{r, 1}^{p s} \\
\tilde{z}_{r, W}^{p s}
\end{array}\right\}=Z_{W}\left(z_{r}^{p s}-z_{1}^{p s}\right)
\end{aligned}
$$

For a SD satellite pair $p s$ and a network station $r$, the network vector of IRC/DSC-estimable parameters is thus given as

$x_{I R C / D S C}=\left[\Delta \tilde{x}_{r}^{T}, d \tilde{t}^{p s},\left[\delta \tilde{t}_{, I F}^{p s}, \tilde{\delta}_{, W}^{p s}\right],\left[\tilde{z}_{r, 1}^{p s}, \tilde{z}_{r, W}^{p s}\right]\right]^{T}$

\subsubsection{User model}

The PPP-RTK corrections of the IRC/DSC-model are given as

$x_{I R C / D S C}^{c o r r}=\left[d \tilde{t}^{p s},\left[\delta \tilde{t}_{, I F}^{p s}, \tilde{\delta}_{, W}^{p s}\right]\right]^{T}$

The correction $\delta \tilde{t}_{, I F}^{p s}$ is called the integer recovery phase clock by Laurichesse and Mercier (2007) and the decoupled phase clock by Collins (2008). The correction $\tilde{\delta}_{, W}^{p s}$ denotes the wide-lane, between-satellite differenced estimable phase-bias.

With the IRC/DSC-corrected observations

$$
\begin{aligned}
\Delta \tilde{\phi}_{u, I F}^{p s} & =\Delta \phi_{u, I F}^{p s}+\delta \tilde{t}_{I F}^{p s} \\
\Delta \tilde{p}_{u, I F}^{p s} & =\Delta p_{u, I F}^{p s}+d \tilde{t}^{p s} \\
\Delta \tilde{\phi}_{u, W N}^{p s} & =\Delta \phi_{u, W N}^{p s}+\lambda_{W} \tilde{\delta}_{W}^{p s}
\end{aligned}
$$

the IRC/DSC user equations take the form

$$
\begin{aligned}
\Delta \tilde{\phi}_{u, I F}^{p s} & =\Delta \tilde{\rho}_{u}^{p s}+\lambda_{N} \tilde{z}_{u, 1}^{p s}+\frac{\lambda_{2}}{\mu_{12}} \tilde{z}_{u, W}^{p s} \\
\Delta \tilde{p}_{u, I F}^{p s} & =\Delta \tilde{\rho}_{u}^{p s} \\
\Delta \tilde{\phi}_{u, W N}^{p s} & =\lambda_{W} \tilde{z}_{u, W}^{p s}
\end{aligned}
$$

The equations (34) and (39) are the between-satellite SD network- and user-equations of the IRC/DSC-model. The equations of (34) are the same as those introduced in Collins et al (2008, p. 1316), be it that in that contribution $\lambda_{N} \tilde{z}_{u, 1}^{p s}+\frac{\lambda_{2}}{\mu_{12}} \tilde{z}_{u, W}^{p s}$ is explictly expressed for the GPS frequency bands only, namely as

$$
\lambda_{I}\left(17 \tilde{z}_{u, 1}^{p s}+60 \tilde{z}_{u, W}^{p s}\right), \quad \lambda_{I}=\frac{2 c f_{o}}{f_{1}^{2}-f_{2}^{2}}
$$

with $f_{o}=10.23 \mathrm{MHz}$ and $c$ being the velocity of light. Similarly, the equations of (34) are the SD version of those given in Eqs. (3) and (5) of Laurichesse et al $(2009$, p. 136, 137), see also Eqs. (4), (5) and (6) of Loyer et al (2012). The only difference lies in further accounting for the phase center offsets in the first two IF observation equations. This difference is, however, neglected once the estimable wide-lane satellite phase biases are to be determined, see e.g. Eq. (4) in Laurichesse et al (2009, p. 136) or Eq. (3) in Loyer et al (2012, p. 993).

\subsubsection{The CC-1, DC, and IRC/DSC Models Compared}

We derived the IRC/DSC-model from the full-rank CCmodel (9), by first formulating the ionosphere-free variant of the CC-model and then applying the one-to-one CC-to-IRC/DSC parameter transformation.

The $\mathcal{S}$-basis that we used is $\Delta x_{1}, d^{p s}, z_{1}^{p s}$ (cf. 8). In Collins (2008), it is stated however, that the $\mathcal{S}$ basis of the DSC-model is given by the ionospherefree and narrow-lane code biases, $d_{, I F}^{p s}=\mu_{I F}^{T} d^{p s}$ and $d_{, N L}^{p s}=\mu_{N L}^{T} d^{p s}$, and the ambiguities $z_{1,1}^{p s}$ and $z_{1, W}^{p s}$. Hence, one may think that for the DSC-model in Collins (2008) a different $\mathcal{S}$-basis choice is made than for the CC- or DC-models, (9) and (17), respectively. This is not true however. As the transformation between $d^{p s}$ and $d_{, I F}^{p s}, d_{, N L}^{p s}$ is one-to-one, and also the transformation between $z_{1}^{p s}$ and $z_{1,1}^{p s}, z_{1, W}^{p s}$ is one-to-one, the DSCchoice in Collins (2008) is identical to the $\mathcal{S}$-basis choice of (9) and (17), respectively, namely $\Delta x_{1}, d^{p s}, z_{1}^{p s}$.

We have already shown, with (31) and (32), that the estimable parameters of the IRC/DSC-model stand in one-to-one correspondence with the estimable parameters of the CC-model (9). Additionally, they also stand in one-to-one correspondence with their DC-model counterparts. The estimable IRC/DSC-parameters can namely be expressed in the estimable DC-parameters as

$$
\begin{aligned}
\Delta \tilde{x}_{r} & =\Delta \tilde{x}_{r} \\
\delta \tilde{t}_{I F}^{p s} & =\frac{\mu_{2}}{\mu_{12}} \delta \tilde{t}_{, 1}^{p s}-\frac{\mu_{1}}{\mu_{12}} \delta \tilde{t}_{, 2}^{p s} \\
d \tilde{t}^{p s} & =d \tilde{t}^{p s} \\
\tilde{\delta}_{, W}^{p s} & =\frac{1}{\lambda_{1}} \delta \tilde{t}_{, 1}^{p s}-\frac{1}{\lambda_{2}} \delta \tilde{t}_{, 2}^{p s}-\frac{1}{\lambda_{W}} d \tilde{t}^{p s} \\
\tilde{z}_{r, 1}^{p s} & =\tilde{z}_{r, 1}^{p s} \\
\tilde{z}_{r, W}^{p s} & =\tilde{z}_{r, 1}^{p s}-\tilde{z}_{r, 2}^{p s}
\end{aligned}
$$

This shows, for instance, that the decoupled phase clock of the IRC/DSC model, $\delta \tilde{t}_{I F}^{p s}$, is simply the ionospherefree version of de Jonge's distinct phase clock of the DC-model, $\delta \tilde{t}_{I F}^{p s}=\mu_{I F}^{T} \delta \tilde{t}^{p s}$.

From the comparison of the CC-1, DC and IRC/DSC models, one can thus conclude that the IRC/DSC-model 
is a reparametrized form of both the DC-model (17) and the CC-model (9) using ionosphere-free observations.

\subsection{The UPD/FCB model}

Just like the IRC/DSC-model is obtained as a reparametrized version of the CC-model (9) using ionosphere-free observations, the UPD/FCB-model can be obtained as a reparametrized version of the CC-model (21) using ionosphere-free observations. In case of the UPD/FCBmodel, the reparametrization is even simpler as it only involves the wide-lane transformation.

\subsubsection{Network Model}

To derive the UPD/FCB network equations, we first form the ionosphere-free variant of (21) and then apply the wide-lane parameter transformation.

If we apply the full-rank $4 \times 3$ matrix of $(29)$ as transformation to (21) to form its three ionosphere-free observation equations, we obtain

$$
\begin{aligned}
\Delta \phi_{r, I F}^{p s} & =\Delta \tilde{\rho}_{r}^{p s}-d \tilde{t}^{p s}+\mu_{I F}^{T} \Lambda \tilde{a}_{r}^{p s} \\
\Delta p_{r, I F}^{p s} & =\Delta \tilde{\rho}_{r}^{p s}-d \tilde{t}^{p s} \\
\Delta \phi_{r, W N}^{p s} & =\quad+\mu_{W L}^{T} \Lambda \tilde{a}_{r}^{p s}
\end{aligned}
$$

With the wide-lane parametrization $\tilde{a}_{r}^{p s}=Z_{W}^{-1}\left[\tilde{a}_{r, 1}^{p s}, \tilde{a}_{r, W}^{p s}\right]^{T}$, thereby making use of (33), the full-rank network equations of the UPD/FCB-model follow as

$$
\begin{aligned}
\Delta \phi_{r, I F}^{p s} & =\Delta \tilde{\rho}_{r}^{p s}-d \tilde{t}^{p s}+\lambda_{N} \tilde{a}_{r, 1}^{p s}+\frac{\lambda_{2}}{\mu_{12}} \tilde{a}_{r, W}^{p s} \\
\Delta p_{r, I F}^{p s} & =\Delta \tilde{\rho}_{r}^{p s}-d \tilde{t}^{p s} \\
\Delta \phi_{r, W N}^{p s} & =\lambda_{W} \tilde{a}_{r, W}^{p s}
\end{aligned}
$$

Note, although the structure of these equations resembles that of the corresponding IRC/DSC equations (34), that the ambiguities in (34) are integer, whereas in (43) they are not.

For a SD satellite pair $p s$ and a network station $r$, the network vector of FCB-estimable parameters is thus given as

$x_{F C B}=\left[\left(\Delta \tilde{x}_{r}\right)^{T}, d \tilde{t}^{p s},\left[\tilde{a}_{r, 1}^{p s}, \tilde{a}_{r, W}^{p s}\right]\right]^{T}$

The interpretation of these estimable parameters is as given earlier for the CC-model (21), be it that the ambiguities are now in wide-lane form. Hence, in contrast to the ambiguities of the ionosphere-free IRC/DSCmodel (cf. 34, 39), the ambiguities of the ionospherefree UPD/FCB-model are not integer-valued, but realvalued.

\subsubsection{User Model}

As in case of the CC-model (21), next to the estimable satellite clock $d \tilde{t}^{p s}$, the real-valued estimable ambiguities (but now in wide-lane form) of any network station, say $q \in\{1, \ldots, n\}$, can be taken to form the corrections for the user,

$x_{F C B}^{c o r r}=\left[d \tilde{t}^{p s},\left[\tilde{a}_{q, 1}^{p s}, \tilde{a}_{q, W}^{p s}\right]\right]^{T}$

With the user ionosphere-free observations corrected as

$\Delta \tilde{\tilde{\phi}}_{u, I F}^{p s}=\Delta \phi_{u, I F}^{p s}+d \tilde{t}^{p s}-\lambda_{N} \tilde{a}_{q, 1}^{p s}-\frac{\lambda_{2}}{\mu_{12}} \tilde{a}_{q, W}^{p s}$

$\underset{\sim}{\sim} \tilde{\tilde{\tilde{p}}}_{u, I F}^{p s}=\Delta p_{u, I F}^{p s}+d \tilde{t}^{p s}$

$\Delta \tilde{\tilde{\phi}}_{u, W N}^{p s}=\Delta \phi_{u, W N}^{p s}-\lambda_{W} \tilde{a}_{q, W}^{p s}$

the user equations take the form

$$
\begin{aligned}
\Delta \tilde{\tilde{\phi}}_{u, I F}^{p s} & =\Delta \tilde{\rho}_{u}^{p s}+\lambda_{N} \tilde{a}_{q u, 1}^{p s}+\frac{\lambda_{2}}{\mu_{12}} \tilde{a}_{q u, W}^{p s} \\
\Delta \tilde{\tilde{p}}_{u, I F}^{p s} & =\Delta \tilde{\rho}_{u}^{p s} \\
\tilde{\tilde{\phi}}_{u, W N}^{p s} & =\lambda_{W} \tilde{a}_{q u, W}^{p s}
\end{aligned}
$$

with the integer double-differenced ambiguities $\tilde{a}_{q u, 1}^{p s}=$ $z_{u, 1}^{p s}-z_{q, 1}^{p s}$ and $\tilde{a}_{q u, W}^{p s}=z_{u, W}^{p s}-z_{q, W}^{p s}$. Note that these user-equations are identical to the user-equations of the IRC/DSC-model (39), be it that their integer doubledifferenced ambiguities could differ in their choice of reference station ( $q$ vs 1 ).

\subsubsection{The fractional phase bias (FPB) corrections}

Since any integer shift of the real-valued ambiguities in (45) would only result in an integer shift of the corresponding ambiguities in the user equations, the PPP-RTK corrections remain admissible if the realvalued ambiguities of (45) are replaced by their fractional parts,

$x_{F P B}^{c o r r}=\left[d \tilde{t}^{p s},\left[\operatorname{frac}\left(\tilde{a}_{q, 1}^{p s}\right), \operatorname{frac}\left(\tilde{a}_{q, W}^{p s}\right)\right]\right]^{T}$

with the fractional operator defined as $\operatorname{frac}(x)=x-$ $\lfloor x\rceil$ (Geng, 2011), where $\lfloor x\rceil$ denotes rounding to the nearest integer of $x$.

Recall from (23) the relation $\tilde{a}_{r}^{p s}=\tilde{z}_{r}^{p s}-\tilde{\delta}^{p s}$. After applying the wide-lane transformation $Z_{W}$, this relation becomes,

$\tilde{a}_{r, 1}^{p s}=\tilde{z}_{r, 1}^{p s}-\tilde{\delta}_{, 1}^{p s}$

$\tilde{a}_{r, W}^{p s}=\tilde{z}_{r, W}^{p s}-\tilde{\delta}_{, W}^{p s}$

Since both $\tilde{z}_{r, 1}^{p s}$ and $\tilde{z}_{r, W}^{p s}$ are integer, it follows that

$\operatorname{frac}\left(\tilde{a}_{q, 1}^{p s}\right)=-\operatorname{frac}\left(\tilde{\delta}_{, 1}^{p s}\right)$

$\operatorname{frac}\left(\tilde{a}_{q, W}^{p s}\right)=-\operatorname{frac}\left(\tilde{\delta}_{, W}^{p s}\right)$

This shows that, apart from their sign, the fractional parts of the real-valued ambiguities are equal to the 
fractional parts of the phase biases. This is the reason why the set of network- and user-equations, (43) and (47), combined with the correction vector (48), is referred to as the Fractional Cycle Bias (FCB) model (Geng et al, 2010, p. 569). The non-integer phase bias $\tilde{\delta}^{p s}$ is also referred to as the 'Uncalibrated Phase Delay' (UPD) by Ge et al (2008, p. 389). The network- and user-equations, (43) and (47), together with the corrections (45), i.e. without the use of the fractional operator, is the formulation used by Bertiger et al (2010).

\subsubsection{The FCB corrections}

The UPD/FCB method (Ge et al, 2008; Geng et al, 2012) uses a somewhat different version of (43). Using the identity $\lambda_{2} / \mu_{12}=\lambda_{N} \lambda_{1} /\left(\lambda_{2}-\lambda_{1}\right)$, we may write $\lambda_{N} \tilde{a}_{r, 1}^{p s}+\frac{\lambda_{2}}{\mu_{12}} \tilde{a}_{r, W}^{p s}=\frac{\lambda_{2}}{\mu_{12}}\left\lfloor\tilde{a}_{r, W}^{p s}\right\rceil+\lambda_{N} \tilde{a}_{r, c}^{p s}$, where

$$
\begin{aligned}
\tilde{a}_{r, c}^{p s} & =\tilde{a}_{r, 1}^{p s}+\frac{\lambda_{1}}{\lambda_{2}-\lambda_{1}} \operatorname{frac}\left(\tilde{a}_{r, W}^{p s}\right) \\
& =\tilde{z}_{r, 1}^{p s}-\tilde{\delta}_{, c}^{p s}
\end{aligned}
$$

with

$\tilde{\delta}_{, c}^{p s}=\tilde{\delta}_{, 1}^{p s}+\frac{\lambda_{1}}{\lambda_{2}-\lambda_{1}} \operatorname{frac}\left(\tilde{\delta}_{, W}^{p s}\right)$

Hence, (43) can alternatively be expressed as

$$
\begin{aligned}
\Delta \phi_{r, I F}^{p s} & =\Delta \tilde{\rho}_{r}^{p s}-d \tilde{t}^{p s}+\frac{\lambda_{2}}{\mu_{12}}\left\lfloor\tilde{a}_{r, W}^{p s}\right\rceil+\lambda_{N} \tilde{a}_{r, c}^{p s} \\
\Delta p_{r, I F}^{p s} & =\Delta \tilde{\rho}_{r}^{p s}-d \tilde{t}^{p s} \\
\Delta \phi_{r, W N}^{p s} & =\lambda_{W} \tilde{a}_{r, W}^{p s}
\end{aligned}
$$

This leads to the use of the following PPP-RTK corrections

$x_{F C B}^{c o r r}=\left[d \tilde{t}^{p s},\left[\operatorname{frac}\left(\tilde{a}_{q, c}^{p s}\right), \operatorname{frac}\left(\tilde{a}_{q, W}^{p s}\right)\right]\right]^{T}$

rather than those given in (48). The user observations would then be corrected as

$$
\begin{aligned}
\Delta \tilde{\tilde{\phi}}_{u, I F}^{\prime p s} & =\Delta \phi_{u, I F}^{p s}+d \tilde{t}^{p s}-\lambda_{N} \operatorname{frac}\left(\tilde{a}_{q, c}^{p s}\right) \\
\Delta \tilde{\tilde{p}}_{u, I F}^{\prime p s} & =\Delta p_{u, I F}^{p s}+d \tilde{t}^{p s} \\
\tilde{\tilde{\phi}}_{u, W N}^{p s} & =\Delta \phi_{u, W N}^{p s}-\lambda_{W} \operatorname{frac}\left(\tilde{a}_{q, W}^{p s}\right)
\end{aligned}
$$

These equations show a somewhat more symmetric form than those of (46). Using the definition of the fractional operator, the user observation equations take then the form (compare with (47))

$$
\begin{aligned}
\Delta \tilde{\tilde{\phi}}_{u, I F}^{\prime p s} & =\Delta \tilde{\rho}_{u}^{p s}+\lambda_{N} a_{u, 1}^{\prime p s}+\frac{\lambda_{2}}{\mu_{12}} a_{u, W}^{\prime p s} \\
\Delta \tilde{\tilde{p}}_{u, I F}^{\prime p s} & =\Delta \tilde{\rho}_{u}^{p s} \\
\tilde{\tilde{\phi}}_{u, W N}^{\prime p s} & =\lambda_{W} a_{u, W}^{\prime p s}
\end{aligned}
$$

with the integer-valued ambiguities

$$
\begin{aligned}
& a_{u, 1}^{\prime p s}=\tilde{a}_{q u, 1}^{p s}+\left\lfloor\tilde{a}_{q, c}^{p s}\right\rceil \\
& a_{u, W}^{\prime p s}=\tilde{a}_{q u, W}^{p s}+\left\lfloor\tilde{a}_{q, W}^{p s}\right\rceil
\end{aligned}
$$

The equations of (53), with (49), are the SD versions of those given in Eqs. (5) and (14) of Ge et al (2008, p. 391), see also Eqs. (3) and (5) of Geng and Bock (2013, p. 451). In those contributions however, the following equivalent expressions are used instead

$\lambda_{N}=\frac{\lambda_{1} f_{1}}{f_{1}+f_{2}}=\frac{c}{f_{1}+f_{2}} ; \quad \frac{\lambda_{2}}{\mu_{12}}=\frac{\lambda_{1} f_{1} f_{2}}{f_{1}^{2}-f_{2}^{2}}$

We already remarked earlier that the ambiguity $\tilde{a}_{r, 1}^{p s}$ is sometimes erroneously referred to as the narrow-lane ambiguity because of its $\lambda_{N}$-coefficient in the observation equations (cf. 34). Similarly, it should be understood that the reference to the fractional part of $\tilde{a}_{q, c}^{p s}$ in (53) as the narrow-lane FCB (Ge et al, 2008; Geng et al, 2010, 2012; Geng and Bock, 2013) follows the same logic.

\subsubsection{The Distribution of the Fractional Phase Bias}

Although an arbitrary integer shift in the PPP-RTK corrections $\tilde{a}_{q}^{p s}$ or $\tilde{\delta}^{p s}$ is of no concern in sofar that it maintains the integerness of the user-ambiguities, it is important to realize that from a probabilistic point of view the application of the fractional operator to the estimated ambiguity $\hat{\tilde{a}}_{q}^{p s}$ or to the estimated satellite phase bias $\hat{\tilde{\delta}}^{p s}$, will change the statistics of the usercorrected observables. In other words, the probability distribution of the user-corrected observations (46) will change from a multivariate normal distribution to a non-normal distribution if one replaces the estimator $\hat{\tilde{a}}_{q}^{p s}$ by its fractional part $\operatorname{frac}\left(\hat{\tilde{a}}_{q}^{p s}\right)=-\operatorname{frac}\left(\hat{\tilde{\delta}}^{p s}\right)$. The following lemma gives the PDF of the fractional phase bias.

Lemma 1 (PDF of fractional phase bias) Let $f_{\hat{\delta}}(x)$ be the probability density function of the satellite phase bias estimator $\hat{\delta}$. Then

$f_{\operatorname{frac}(\hat{\delta})}(x)=\sum_{z \in \mathbb{Z}} f_{\hat{\delta}}(x+z) s_{0}(x)$

with $\mathcal{P}_{0}$ the pull-in region and $s_{0}(x)$ its indicator function $\left(s_{0}(x)=1\right.$ if $x \in \mathcal{P}_{0}, s_{0}(x)=0$ otherwise).

Proof Follows from the GNSS ambiguity residual PDF of Teunissen (2002, p. 44).

For a normally distributed estimated satellite phase bias, the $\operatorname{PDF} f_{\operatorname{frac}(\hat{\delta})}(x)$ is determined by the mean and variance of $\hat{\delta}$. The peakedness of the $\operatorname{PDF} f_{\operatorname{frac}(\hat{\delta})}(x)$ is driven by the variance of $\hat{\delta}$. The larger the variance, the flatter the PDF, which in the limit becomes a uniform distribution over the pull-in region $\mathcal{P}_{0}$. The smaller the variance, the more peaked the PDF becomes, with increasing probability mass becoming concentrated at its mean. 

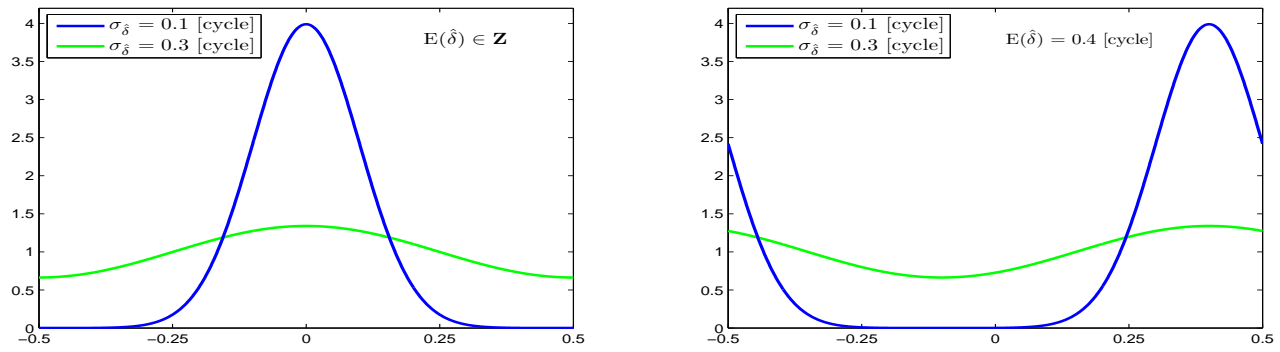

Fig. 1 PDF $f_{\text {frac }(\hat{\delta})}(x)$ of fractional phase bias frac $(\hat{\delta})$ for $\hat{\delta} \sim \mathrm{N}\left(\mathrm{E}(\hat{\delta}), \sigma_{\hat{\delta}}^{2}\right)$. Left: $\mathrm{E}(\hat{\delta}) \in \mathbb{Z}$, with $\sigma_{\hat{\delta}}=0.1$ cycle (blue curve) and $\sigma_{\hat{\delta}}=0.3$ cycle (green curve); Right: $\mathrm{E}(\hat{\delta})=0.4$ cycle, with $\sigma_{\hat{\delta}}=0.1$ cycle (blue curve) and $\sigma_{\hat{\delta}}=0.3$ cycle (green curve).

Would the mean be integer, as is the case with the mean of the float solution of a double-differenced ambiguity, then the PDF of the fraction would be symmetric with respect to the origin. In case of a noninteger mean however, the PDF will be asymmetric with respect to the origin. This is the case that applies to the satellite phase bias.

Figure 1 shows some examples for the scalar case. In the scalar case, $\mathcal{P}_{0}$ is the origin-centred interval of length 1. Figure 1 shows the fractional phase bias PDF $f_{\operatorname{frac}(\hat{\delta})}(x)$ for $\hat{\delta} \sim \mathrm{N}\left(\mathrm{E}(\hat{\delta}), \sigma_{\hat{\delta}}^{2}\right)$. The PDF is shown for two different precision levels of the estimated phase bias, $\sigma_{\hat{\delta}}=0.1$ cycle (blue curve) and $\sigma_{\hat{\delta}}=0.3$ cycle (green curve). The left panel of the figure shows then the corresponding PDFs for the case the mean of the estimated phase bias would be integer, $\mathrm{E}(\hat{\delta}) \in \mathbb{Z}$, while in the right panel the two PDFs are shown for a noninteger mean, $\mathrm{E}(\hat{\delta})=0.4$ cycle.

The above shows that when use is made of the fractional operator, one has to be very careful when evaluating the statistics and quality of the user-corrected observables. As the user-corrected observables will then in principle fail to be normally distributed, it will affect quality control procedures that are applied at the user side. Only in case sufficient probability mass of the normal distribution of $\hat{\delta}$ is located in the pull-in region $\mathcal{P}_{0}$, can one hope to be able to approximate the PDF of $\operatorname{frac}(\hat{\delta})$ by that of $\hat{\delta}$. Whether or not this is the case cannot be judged on only the variance $\sigma_{\hat{\delta}}^{2}$, but requires information about the unknown mean $\mathrm{E}(\hat{\delta})$ as well.

\subsubsection{The CC-2, IRC/DSC and UPD/FCB Models Compared}

We derived the UPD/FCB-model from the full-rank CC-model (21), by first formulating the ionosphere-free variant of the CC-model and then applying the one-toone widelane transformation.
The $\mathcal{S}$-basis that we used is $\Delta x_{1}, d^{p s}, \delta^{p s}$ (cf. 20). In Geng (2011), it is stated however, that the $\mathcal{S}$-basis of the UPD/FCB-model is given by the ionosphere-free and narrow-lane code biases, $d_{, I F}^{p s}=\mu_{I F}^{T} d^{p s}$ and $d_{, N L}^{p s}=$ $\mu_{N L}^{T} d^{p s}$, and the phase biases $\delta_{1}^{p s}, \delta_{1, W}^{p s}$. Hence, one may think that for the UPD/FCB-model in Ge et al (2008) a different $\mathcal{S}$-basis choice is made. This is not true however, as the transformation between $d^{p s}$ and $d_{, I F}^{p s}, d_{, N L}^{p s}$ is one-to-one, and also the wide-lane transformation between $\delta^{p s}$ and $\delta_{1}^{p s}, \delta_{1, W}^{p s}$ is one-to-one. Hence, the $\mathcal{S}$ basis choice is the same, namely $\Delta x_{1}, d^{p s}, \delta^{p s}$.

We already noted that the IRC/DSC user equations (39) are identical to those of UPD/FCB (47) if $q=1$. Their network equations are quite different however, compare (34) for IRC/DSC with (43) for UPD/FCB. How is this possible? The reason lies in their PPP-RTK corrections. Although some of the individual parameters that make up their corrections differ, namely

$\left[\delta \tilde{t}_{, I F}^{p s}, \tilde{\delta}_{, W}^{p s}\right]_{I R C / D S C} v s\left[\operatorname{frac}\left(\tilde{a}_{q, 1}^{p s}\right), \operatorname{frac}\left(\tilde{a}_{q, W}^{p s}\right)\right]_{F C B}$

the actual corrections that the user observations undergo have the same effect. The user observation corrections for the IRC/DSC-model (cf. 38) and the UPD/FCBmodel (cf. 46), satisfy namely

$\delta \tilde{t}_{I F}^{p s}=d \tilde{t}^{p s}-\lambda_{N} \tilde{a}_{q, 1}^{p s}-\frac{\lambda_{2}}{\mu_{12}} \tilde{a}_{q, W}^{p s}$ for $q=1$

$d \tilde{t}^{p s}=d \tilde{t}^{p s}$

$\tilde{\delta}_{W}^{p s}=-\tilde{a}_{q, W}^{p s}$ for $q=1$

while for $q \neq 1$, the corrections only make the userambiguities of the two models differ by an integer shift.

From the comparison of the CC, IRC/DSC, and UPD/FCB models, one can thus conclude that the UPD/FCB-model is a reparametrized form of the ionospherefree version of the CC-model (21), while the IRC/DSCmodel is that of the CC-model (9). An overview of the transformational links between the various models is given in Figure 2. 


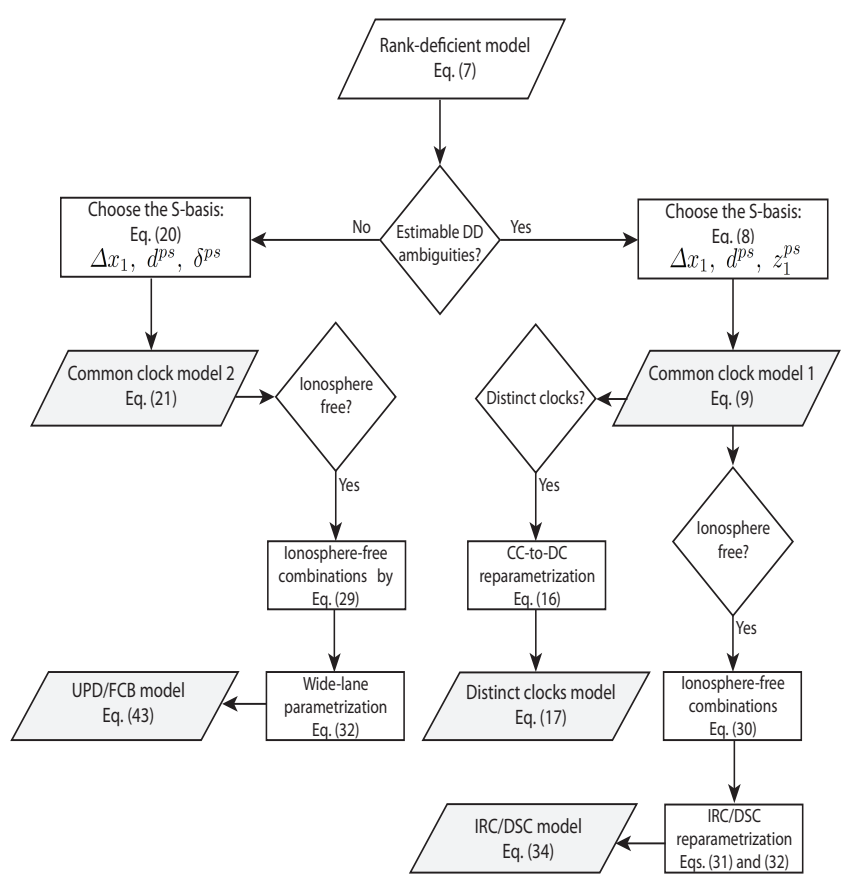

Fig. 2 Diagram illustrating the transformational links between the original rank-deficient model and the PPP-RTK full-rank variants: CC-1 (cf. 9), DC (cf. 17), CC-2 (cf. 21), IRC/DSC (cf. 34), and UPD/FCB (cf. 43).

\section{Role of the PPP-RTK corrections}

In the previous section different formulations of the network- and user models were compared. As they could all be derived from the same model, without any change in underlying model assumptions, all the presented formulations are intrinsically the same. Their differences were shown to lie only in (a) the choice of $\mathcal{S}$-basis, (b) in the choice of parameterization, and (c) in the choice of whether or not to eliminate the ionospheric delays.

Since the different formulations are intrinsically the same, their individual set of PPP-RTK corrections are closely linked. In this section we will have a closer look at these corrections and show how they can be interpreted and transformed.

\subsection{The Same Corrected User Observations}

When we compare the different user-equations, we note that they are essentially the same. This is true for the IRC/DSC and UPD/FCB ionosphere-free user-equations, (39), (47) and (56), since they only differ in a possible integer-shift of their ambiguities. The same holds true for the two sets of CC user-equations, (14) and (27).

The sameness of the user-equations implies, that the corrected user-observations themselves are, apart from the presence of integer shifts, also the same. Thus for the two different CC-corrected user observations we have

$\Delta \tilde{\phi}_{u}^{p s} \stackrel{(13,26)}{=} \Delta \tilde{\tilde{\phi}}_{u}^{p s} \pm \Lambda z, \quad z \in \mathbb{Z}^{2}$

$\Delta \tilde{p}_{u}^{p s} \stackrel{(13,26)}{=} \Delta \tilde{\tilde{p}}_{u}^{p s}$

while for the ionosphere-free, IRC/DSC and UPD/FCB, corrected user-observations we have

$\Delta \tilde{\phi}_{u, I F}^{p s} \stackrel{(38,55)}{=} \Delta \tilde{\tilde{\phi}}_{u, I F}^{p s} \pm \lambda_{N}\left(z_{1}+\frac{\lambda_{1}}{\lambda_{2}-\lambda_{1}} z_{W}\right), z_{1}, z_{W} \in \mathbb{Z}$

$\Delta \tilde{p}_{u, I F}^{p s} \stackrel{(38,55)}{=} \Delta \tilde{\tilde{p}}_{u, I F}^{p s}$

$\Delta \tilde{\phi}_{u, W N}^{p s} \stackrel{(38,55)}{=} \Delta \tilde{\tilde{\phi}}_{u, W N}^{\prime p s} \pm \lambda_{W} z_{W}$

Since BLUE-estimation, i.e. properly weighted leastsquares estimation, is intrinsically invariant for differences in choice of (a) $\mathcal{S}$-basis, (b) parameterization, and (c) whether or not some parameters, like ionospheric delays, are eliminated, the application of a rigorous network adjustment, using any one of the different PPPRTK methods, must give the same positioning results for the user. Any differences that show up between the results of the different PPP-RTK methods, must then be attributed to the usage of a different nonrigorous estimation procedure. We will revisit this remark in section 6.2 .

\subsection{Transformation between PPP-RTK Corrections}

As the user-equations of the user-corrected observations are essentially the same, the different PPP-RTK corrections must contain the same information and hence be related through one-to-one transformations. We have summarized these transformations in Table 1.

For example, if one would like to transform from the IRC/DSC corrections to the CC-1 corrections, then the following transformation applies:

$\left.\left[\begin{array}{l}d \tilde{t}^{p s} \\ \tilde{\delta}_{, 1}^{p s} \\ \tilde{\delta}_{, 2}^{p s}\end{array}\right]_{\mathrm{CC}-1}=\left[\begin{array}{ccc}1 & 0 & 0 \\ -\frac{1}{\lambda_{N}} & \frac{1}{\lambda_{N}}-\frac{\lambda_{1}}{\lambda_{2}-\lambda_{1}} \\ -\frac{1}{\lambda_{N}} & \frac{1}{\lambda_{N}}-\frac{\lambda_{2}}{\lambda_{2}-\lambda_{1}}\end{array}\right]_{, W}\right]_{\mathrm{IRC} / \mathrm{DSC}}$

These transformations can now be used to operationally link the different PPP-RTK methods and to mix them between network and users. It allows a user to transform the network received corrections to the format that suits his/her user software, or alternatively, as a service to the users, it allows a network server to transform the network corrections to any one of the other formats and thus make any of the different PPPRTK parametrizations available to the users.

\subsection{Interpretation of the PPP-RTK corrections}

The PPP-RTK corrections establish a link between the user-parameters and the network-parameters. The corrections are designed to realize integer ambiguities in 
Table 1 PPP-RTK user-corrections in different models

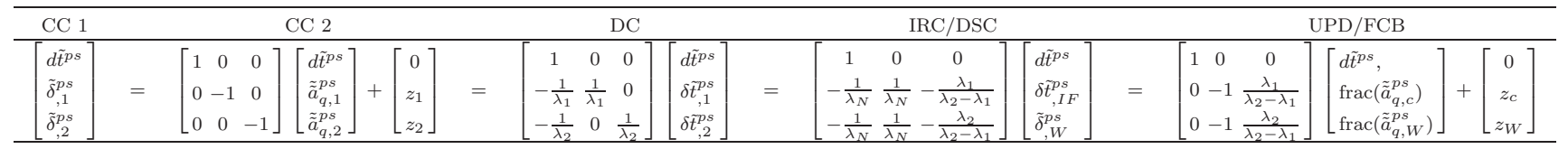

The integers $z_{1}, z_{2}, z_{c}, z_{W}$ may take any integer values

the user-equations, thus enabling user integer ambiguity resolution. As remarked earlier, the interpretation of these 'user' integer ambiguities is that they are straightforward double differenced (DD) ambiguities.

The presence of $z_{1}^{p s}$ in the phase bias correction vector of CC-1,

$\tilde{\delta}^{p s}=\delta^{p s}+M d^{p s}-z_{1}^{p s}$

or, in the real-valued ambiguity correction vector of CC2 ,

$\tilde{a}_{1}^{p s}=z_{1}^{p s}-\delta^{p s}-M d^{p s}$

or, in the integer recovery clock and wide-lane phase bias corrections of IRC/DSC,

$\delta \tilde{t}_{I F}^{p s}=d t^{p s}+\mu_{I F}^{T} \Lambda\left(\delta^{p s}-z_{1}^{p s}\right)-\Delta \rho_{1}^{p s}$

$\tilde{\delta}_{W}^{p s}=(0,1) Z_{W}\left(\delta^{p s}-z_{1}^{p s}\right)-\frac{1}{\lambda_{W}} \mu_{N L}^{T} d^{p s}$

make that the user-ambiguities in all these cases become DD ambiguities between user station $u$ and network station 1.

Next to establishing an ambiguity link between network and user, the PPP-RTK corrections also establish a positional link between network and user. The presence of $\Delta \rho_{1}^{p s}$ in the satellite clock correction

$d \tilde{t}^{p s}=d t^{p s}+\mu_{I F}^{T} d^{p s}-\Delta \rho_{1}^{p s}$

and in the integer recovery clock correction $\delta \tilde{t}_{I F}^{p s}$ in case of the IRC/DSC method, makes that the userpositioning parameters in essence become relative positioning parameters between user station $u$ and network station 1. The PPP-RTK method is thus a relative positioning method and not one of absolute positioning.

To demonstrate the relative positioning feature of PPP-RTK, consider the special case that the network consists of only one single station, i.e. $n=1$. It then follows from (9), since $\Delta \tilde{\rho}_{1}^{p s}=0\left(\Delta \tilde{x}_{1}=0\right)$ and $\tilde{z}_{1}=0$, that

$$
\begin{aligned}
& \Delta \phi_{1}^{p s}=-\mu \tilde{\iota}_{1}^{p s}-e d \tilde{t}^{p s}-\Lambda \tilde{\delta}^{p s} \\
& \Delta p_{1}^{p s}=+\mu \tilde{\iota}_{1}^{p s}-e d \tilde{t}^{p s}, \quad s=1, \ldots, m
\end{aligned}
$$

This is an invertible system of $4(m-1)$ equations in $4(m-1)$ unknowns. After inversion, we get

$$
\begin{aligned}
\tilde{\iota}_{1}^{p s} & =+\mu_{G F}^{T} \Delta p_{1}^{p s} \\
d \tilde{t}^{p s} & =-\mu_{I F}^{T} \Delta p_{1}^{p s} \\
\tilde{\delta}^{p s} & =-\Lambda^{-1}\left[\Delta \phi_{1}^{p s}+\left(\mu \mu_{G F}^{T}-e \mu \mu_{I F}^{T}\right) \Delta p_{1}^{p s}\right]
\end{aligned}
$$

Substitution of these expressions for $d \tilde{t}^{p s}$ and $\tilde{\delta}^{p s}$ into (13) gives the user observation equations as

$$
\begin{aligned}
& \Delta \tilde{\phi}_{u}^{p s}=\Delta \phi_{1 u}^{p s}-\mu \mu_{G F}^{T} \Delta p_{1}^{p s}=e \Delta \tilde{\rho}_{u}^{p s}-\mu \tilde{\iota}_{u}^{p s}+\Lambda \tilde{z}_{u}^{p s} \\
& \Delta \tilde{p}_{u}^{p s}=\Delta p_{1 u}^{p s}+\mu \mu_{G F}^{T} \Delta p_{1}^{p s}=e \Delta \tilde{\rho}_{u}^{p s}+\mu \tilde{\iota}_{u}^{p s}
\end{aligned}
$$

in which we recognize the DD phase and code obervations, $\Delta \phi_{1 u}^{p s}=\Delta \phi_{u}^{p s}-\Delta \phi_{1}^{p s}$ and $\Delta p_{1 u}^{p s}=\Delta p_{u}^{p s}-\Delta p_{1}^{p s}$, respectively.

This demonstrates that the PPP-RTK corrected observations are actually DD observations biased with an additional iono-term ' $\mu \mu_{G F}^{T} \Delta p_{1}^{p s}$. This bias term is of no consequence, since it gets fully absorbed in the ionospheric term $\tilde{\iota}_{u}^{p s}$. It does therefore not affect the solution for $\Delta \tilde{x}_{u}$ (or $\Delta \tilde{\rho}_{u}^{p s}$ ) and $\tilde{z}_{u}^{p s}$. Hence, the PPP-RTK generated solution of the above user model (71) is indeed a relative positioning solution, and one, that will be identical to a standard DD single baseline solution.

In fact, in its ionosphere-free formulation, the bias ' $\mu \mu_{G F}^{T} \Delta p_{1}^{p s}$ ' is eliminated and the observables will consist solely of DD combinations,

$$
\begin{aligned}
\Delta \tilde{\phi}_{u, I F}^{p s} & =\Delta \phi_{1 u, I F}^{p s} \\
\Delta \tilde{p}_{u, I F}^{p s} & =\Delta p_{1 u, I F}^{p s} \\
\Delta \tilde{\phi}_{u, W N}^{p s} & =\Delta \phi_{1 u, W N}^{p s}
\end{aligned}
$$

Thus the only difference between the above PPP-RTK solution and a standard DD single baseline solution is that with (71) a between-satellite SD ionospheric delay $\tilde{\iota}_{u}^{p s}$ is estimated, while in the DD single baseline case, with the absence of ' $\mu \mu_{G F}^{T} \Delta p_{1}^{p s}$, this would be the DD ionospheric delay $\tilde{\iota}_{1 u}^{p s}=\tilde{\iota}_{u}^{p s}-\tilde{\iota}_{1}^{p s}$.

As to the number of correction parameters required, we note that the PPP-RTK method only requires the $3(m-1)$ corrections $d \tilde{t}^{p s}$ and $\tilde{\delta}^{p s}$, while a standard DD approach requires all original $4(m-1)$ phase and code data $\Delta \phi_{1}^{p s}$ and $\Delta p_{1}^{p s}$.

\subsection{On the Ionosphere-free Formulation}

One may wonder what the benefits are of using an ionosphere-free formulation? Surely this is not an improved quality of its solution, since, when rigorously solved, the ionosphere-free model formulations of Section 3 give exactly the same solution as when using 
the original model formulations of Section 2. An apparent benefit of this traditional ionosphere-free formulation is that less parameters need to be solved for as all ionospheric delays have been eliminated. But still, if one is not interested in these ionosphere delays, then it is really not needed to have them a priori eliminated from the model. If one wants them eliminated, one can simply reduce the normal equations for these ionospheric delays. This has the advantage that one can still work with the original, usually uncorrelated, observations and their equations, instead of with the correlated ionosphere-free observations.

We find that the ionosphere-free formulation, instead of having clear benefits, has the drawback of lacking flexibility for further model strengthening, see e.g. Teunissen and de Bakker (2012) or Mervart et al (2013). The inclusion of a dynamic state transition model to capture the temporal smoothness of the ionosphere, for instance, will be problematic with the ionosphere-free model. A similar difficulty exists when one wants to incorporate an ionospheric model to capture both the temporal and spatial characteristics of the ionosphere.

It is well-known that the ionosphere-free or ionosphere-float models are relatively weak in the sense of their ambiguity resolution capabilities. Relatively long observation time spans are then needed to achieve successful integer ambiguity resolution (Hernandez-Pajares et al, 2000; Jonkman et al, 2000; Odijk, 2002; Odijk et al, 2014a). The lack of any ionospheric information is, in fact, the bottleneck for fast ambiguity resolution. Successful ambiguity resolution is achieved much faster when such ionospheric information can be provided to the model. But if such information would be available, it would be cumbersome to include in the ionosphere-free model. Doing so, would also defy the whole purpose of an ionosphere-free formulation. This is straightforward, however, with our original model formulations, like (9) or (21), on the network side, and (14) or (27), on the user side. On the network side, for instance, the inclusion of an ionospheric model would result in a strengthening of the model through the parametrization of the slant delays $\tilde{\iota}_{r}^{p s}$ into fewer parameters. And on the userside, the model would be strengthened through the provision of a priori information on the ionospheric delays of the user, as was already demonstrated in the PPPRTK concept of Teunissen et al (2010) and Odijk et al (2012, 2014b).

In order for the PPP-RTK concept to be better applicable to a wider range of network and user station separations (i.e., from close to distant), the corrections will have to include ionospheric information. Consider, for instance, the case where BLUP (i.e., least-squares interpolation) is used to predict the ionospheric user de- lay, say $\tilde{\iota}^{p s}$, from the network delays $\tilde{\imath}_{r}^{p s}, r=1, \ldots, n$. Then, next to applying $d \tilde{t}^{p s}$ and $\tilde{\delta}^{p s}$, an application of the ionosphere correction gives for the user equations,

$$
\begin{aligned}
& \Delta \phi_{u}^{p s}+\mu \tilde{\iota}^{p s}+e d \tilde{t}^{p s}+\Lambda \tilde{\delta}^{p s}=e \tilde{\rho}_{u}^{p s}-\mu\left(\tilde{\iota}_{u}^{p s}-\tilde{\iota}^{p s}\right)+\Lambda \tilde{z}_{u}^{p s} \\
& \Delta p_{u}^{p s}-\mu \tilde{\iota}^{p s}+e d \tilde{t}^{p s}=e \tilde{\rho}_{u}^{p s}+\mu\left(\tilde{\iota}_{u}^{p s}-\tilde{\iota}^{p s}\right)
\end{aligned}
$$

Hence, at the user-side the biased ionospheric slant delay $\tilde{\iota}_{u}^{p s}$ is now replaced with its difference to $\tilde{\iota}^{p s}$. This is a flexible formulation as it allows one to a priori weigh the difference $\tilde{\iota}_{u}^{p s}-\tilde{\iota}^{p s}$ in accordance to the ionospheric prediction error. The smaller the prediction error, the stronger the model and the larger the ambiguity success rate. In the limit one would have $\tilde{\iota}^{p s}=\tilde{\iota}_{u}^{p s}$, thus providing a strength that is equivalent to that of an ionosphere-fixed, short baseline model.

\section{Estimation of the PPP-RTK corrections}

In this section we discuss 4 different least-squares estimators of the PPP-RTK corrections: the float and fixed estimators under the geometry-free (GF) model and the float and fixed estimators under the geometrybased (GB) model. The different characteristics of these estimators will also facilitate our discussion of some of the estimation approaches described in the literature.

As the previous sections have shown, there are different forms of network equations one can start from. Hence, the four mentioned estimators can be determined for any of these network equations. The CCmodel (9) would have our preference, as its observation equations are already parameterized in integer ambiguities. However, to make an easier link with some of the other approaches, as well as to show how integer ambiguity resolution can be incorporated when the estimated ambiguities are non-integer, we start here from the network equations of the CC-model (21). With this CC-2 model we discuss the four different estimators for $d \tilde{t}^{p s}$ and $\tilde{a}_{q}^{p s}$. The corresponding estimators of any of the other forms of corrections can then easily be obtained through the transformations given in Table 1

\subsection{Geometry-free Estimation}

If we define $\phi_{r}=\left[\phi_{r, 1}^{T}, \phi_{r, 2}^{T}\right]^{T} \in \mathbb{R}^{2 m}, \phi_{r, j}=\left[\Delta \phi_{r, j}^{1}, \ldots,-\right.$ $\left.\Delta \phi_{r, j}^{m}\right]^{T}, j=1,2$, with a likewise definition for $p_{r}$, we can obtain from (21), in vector-matrix form, a uniquely solvable set of equations for station $r$,

$$
\mathrm{E}\left[\begin{array}{c}
\left(I_{2} \otimes D_{m}^{T}\right) \phi_{r} \\
\left(I_{2} \otimes D_{m}^{T}\right) p_{r}
\end{array}\right]=\left[\begin{array}{c}
(-\mu,-e, \Lambda) \otimes I_{m-1} \\
(+\mu,-e, 0) \otimes I_{m-1}
\end{array}\right]\left[\begin{array}{c}
\tilde{\iota}_{r} \\
d \tilde{t}_{r} \\
\tilde{a}_{r}
\end{array}\right]
$$


where $\otimes$ denotes the Kronecker product (Rao, 1973), $D_{m}^{T}$ denotes an $(m-1) \times m$ between-satellite differencing matrix, and $\tilde{\iota}_{r}, d \tilde{t}_{r}$, and $\tilde{a}_{r}$ denote the vectors of $(m-1)$ between-satellite differenced estimable ionospheric delays, satellite clocks and ambiguities of station $r$, respectively. Note that $d \tilde{t}^{p s}$ and $\Delta \tilde{\rho}_{r}^{p s}$ in (21) have been lumped to $d \tilde{t}_{r}^{p s}=d \tilde{t}^{p s}-\Delta \tilde{\rho}_{r}^{p s}$ in $(74)\left(d \tilde{t}_{1}^{p s}=\right.$ $\left.d \tilde{t}^{p s}\right)$. The system (74) is referred to as geometry-free since its design matrix is independent of the receiversatellite geometry (Teunissen, 1997a).

The variance matrix of the observables of (74) is assumed given as

$\mathrm{D}\left[\begin{array}{c}\left(I_{2} \otimes D_{m}^{T}\right) \phi_{r} \\ \left(I_{2} \otimes D_{m}^{T}\right) p_{r}\end{array}\right]=c_{r}^{2}\left[\begin{array}{cc}C_{\phi} & 0 \\ 0 & C_{p}\end{array}\right] \otimes C_{s}$

in which $C_{s}=D_{m}^{T} C_{S} D_{m}$, with $C_{S}$ the co-factor matrix that captures the satellite elevation dependency. The scalar $c_{r}^{2}(r=1, \ldots, n)$ is a receiver-dependent cofactor. In this contribution we assume all receivers of the same quality and thus $c_{r}^{2}=1$ for all $r$. The $2 \times 2$ positive-definite matrices $C_{\phi}$ and $C_{p}$ are the co-factor matrices of the phase and code observable types, respectively.

As the design matrix of (74) is square and invertible, the float solution of $d \tilde{t}_{r}$ and $\tilde{a}_{r}$ is easily obtained.

Lemma 2 (GF float corrections) The geometry-free float solutions $d \hat{\tilde{t}}_{r, G F}$ and $\hat{\tilde{\tilde{a}}}_{r, G F}$ of (74), and their (co)variance matrices are given as

$$
\begin{aligned}
d \hat{\tilde{t}}_{r, G F} & =-\left[\mu_{I F}^{T} \otimes D_{m}^{T}\right] p_{r} \\
\hat{\tilde{a}}_{r, G F} & =+\left[\Lambda^{-1} \otimes D_{m}^{T}\right]\left[\phi_{r}-\left(L \otimes I_{m-1}\right) p_{r}\right]
\end{aligned}
$$

and

$Q_{d \tilde{\hat{t}}_{r} d \hat{\tilde{t}}_{r}}^{G F}=c_{\hat{\rho}}^{2} \otimes C_{s}$

$Q_{d \tilde{\tilde{t}}_{r} \hat{\tilde{a}}_{r}}^{G F}=c_{\hat{\rho}}^{2} e_{\mu}^{T} \Lambda^{-1} \otimes C_{s}$

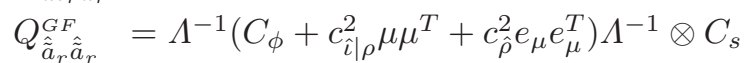

with $L=I_{2}-2 \mu \mu_{G F}^{T}=-[\mu, e][\mu,-e]^{-1}, c_{\hat{\rho}}^{2}=\mu_{I F}^{T} C_{p} \mu_{I F}$, $c_{\hat{\iota} \mid \rho}^{2}=\left(\mu^{T} C_{p}^{-1} \mu\right)^{-1}, e_{\mu}=e-\left(c_{\hat{\rho} \hat{\iota}} / c_{\hat{\rho}}^{2}\right) \mu, c_{\hat{\rho} \hat{\iota}}=\mu_{I F}^{T} C_{p} \mu_{G F}$.

Proof See Appendix.

The variance matrix $Q_{\tilde{\tilde{a}}_{r}}^{G F} \hat{\tilde{a}}_{r}$ has the typical structure of a geometry-free ambiguity variance matrix (Teunissen, 1997c). It has been decomposed such as to clearly show the contribution of the phase and code precision. As shown in the next section, the range-related component that depends on $c_{\hat{\rho}}^{2}$ is the part that will be improved when one switches from the geometry-free model to the geometry-based model.

The GF float solution can be obtained on a stationby-station basis. This is not the case for the ambiguityfixed solution. To the fixed solutions $d \tilde{\tilde{t}}_{r}$ and $\check{\tilde{a}}_{r}$, the data of all network stations contribute. This is a consequence of the fact (cf. 23) that not the station ambiguities $\tilde{a}_{r}$, but the between-station ambiguities $\tilde{a}_{r}-\tilde{a}_{1}=\tilde{z}_{r}$ are integer. Hence, it is these double differences that are estimated as integers and not the between-satellite differenced station ambiguities $\tilde{a}_{r}$. The precision of these estimated station ambiguities, as well as that of the satellite clocks, will benefit however from such integer ambiguity resolution.

The GF ambiguity-fixed solution of the satellite clock $d \tilde{t}_{r}$ and of the real-valued ambiguity vector $\tilde{a}_{r}$ is given in the following lemma.

Lemma 3 (GF fixed corrections) The geometry-free fixed solutions $d \check{\tilde{t}}_{r, G F}$ and $\check{\tilde{a}}_{r, G F}$ of (74), and their (co)variance matrices are given as

$d \check{\tilde{t}}_{r, G F}=d \hat{\tilde{\hat{t}}}_{r, G F}-Q_{d \tilde{\hat{t}}_{r}}^{G F} \hat{\tilde{a}}_{r} Q_{\tilde{\tilde{a}}_{r}}^{G F-1} \hat{\tilde{\tilde{a}}}_{r}\left[\hat{\tilde{a}}_{r, G F}-\check{\tilde{a}}_{r, G F}\right]$

$\check{\tilde{a}}_{r, G F}=\check{\tilde{z}}_{r}+\frac{1}{n} \sum_{j=1}^{n}\left[\hat{\tilde{a}}_{j, G F}-\check{\tilde{z}}_{j}\right]$

and

$Q_{d \tilde{t}_{r} d \check{t}_{r}}^{G F}=Q_{d \tilde{t}_{r} d \hat{\tilde{t}}_{r}}^{G F}-\frac{n-1}{n}\left(c_{\hat{\rho}}^{2}-c_{\tilde{\rho}}^{2}\right) \otimes C_{s} \approx \frac{1}{n} Q_{d \tilde{\hat{t}}_{r} d \hat{\tilde{t}}_{r}}^{G F}$

$Q_{d \tilde{t}_{r} \tilde{\tilde{a}}_{r}}^{G F}=\frac{1}{n} Q_{d \hat{\tilde{t}}_{r}}^{G F} \hat{\tilde{a}}_{r}$

$Q_{\tilde{\tilde{a}}_{r}}^{G F} \check{\tilde{a}}_{r}=\frac{1}{n} Q_{\tilde{\tilde{a}}_{r}}^{G F} \tilde{\tilde{a}}_{r}$

in which $\check{\tilde{z}}_{i}(i=1, \ldots, n)$, with $\check{\tilde{z}}_{1}=0$, are the geometryfree integer resolved DD ambiguities of the network. The cofactor $c_{\tilde{\rho}}^{2}$ is given as $c_{\tilde{\rho}}^{2}=\mu_{I F}^{T}\left(C_{\phi}^{-1}+L^{T} C_{p}^{-1} L\right)^{-1} \mu_{I F}$.

Proof See Appendix.

Note that the difference of the fixed and float solution of $d \tilde{t}_{r}$ only depends on the ambiguity residual of the station $r$ itself. This is due to the fact that $d \hat{\tilde{t}}_{r, G F}$ and $\hat{\tilde{a}}_{q, G F}$ are uncorrelated for $q \neq r$.

Also note, since $\tilde{a}_{r}=\tilde{z}_{r}-\tilde{\delta}$ (cf. 23), that the average in the second equation of (78) is the GF fixed solution of the negative phase bias $-\tilde{\delta}$. Thus

$\check{\tilde{\delta}}_{G F}=-\frac{1}{n} \sum_{j=1}^{n}\left[\hat{\tilde{a}}_{j, G F}-\check{\tilde{z}}_{j}\right]$

with variance matrix

$Q_{\tilde{\tilde{\delta}} \tilde{\tilde{\delta}}}^{G F}=Q_{\tilde{\tilde{a}}_{r}}^{G F} \tilde{\tilde{a}}_{r}=\frac{1}{n} Q_{\tilde{\tilde{a}}_{r}}^{G F} \hat{\tilde{a}}_{r}$

where we assumed the ambiguity success-rate large enough to neglect the uncertainty in the integer solution $\check{\tilde{z}}_{r}$.

We return to these equations later when the FCB approach of estimation is discussed. We remark that the average in (80) will generalize to a weighted-average when the station-dependent factors $c_{r}^{2}$ of (75) would be chosen different from 1 . This would be the case, for 
Table 2 GF and GB multi-epoch redundancy table

\begin{tabular}{|c|c|c|}
\hline Redundancy & float & fixed \\
\hline $\mathrm{GF}$ & $2(m-1)(k-1) n$ & $2(m-1)(k n-1)$ \\
$\mathrm{GB}$ & $\mathrm{GF}+[k(m-1)-\nu](n-1)$ & $\mathrm{GF}+[k(m-1)-\nu](n-1)$ \\
\hline
\end{tabular}

instance, when receivers of different quality would participate in the network.

Lemma 3 shows the GF precision improvement that one can expect to achieve in the clock and ambiguity estimators as a result of successful integer ambiguity resolution. It shows that the gain in precision (approximately) follows the $1 / \sqrt{n}$ rule. For the ambiguities this is due to the network averaging that takes place in computing $\check{\tilde{a}}_{r, G F}$ (cf. 78) and for the satellite clock $d \tilde{\tilde{t}}_{r, G F}$ it follows from using the approximation $c_{\check{\rho}} / c_{\hat{\rho}} \approx 0$ (cf. 79).

This $1 / \sqrt{n}$ improvement, although significant, is not as spectacular as the two orders of magnitude improvement that one achieves in baseline precision when applying instantaneous ambiguity resolution (Teunissen, 1997a). The explanation for this difference lies in the type of parameters considered. As ambiguity resolved single-differenced parameters still require code data for their estimation, the relatively poor code precision prohibits the gain to reach the two orders of magnitude level.

The above results are based on single-epoch solutions. A corresponding multi-epoch solution, based on the time-invariance of $\tilde{a}_{r}$, can subsequently be obtained. We combine these results with our discussion of the geometry-based model in the next section.

\subsection{Geometry-based Estimation}

Let us first consider the redundancies when comparing the GF-model with the GB-model, see Table 2. The single-epoch GF-model has no redundancy. In the $k$ epoch case, however, the time-invariance of the $2(m-1)$ ambiguites of each of the $n$ stations, makes the multirepoch GF-redundancy equal to $2(m-1)(k-1) n$. Would all ambiguities be known, the redundancy would increase further with $2(m-1) n$. However, since ambiguity resolution only affects the integer DD ambiguities, not all, but only $2(m-1)(n-1)$ ambiguities can be considered known. With this increase of redundancy, the ambiguity-fixed, multi-epoch GF-redundancy is equal to $2(m-1)(n k-1)$.

Let us now consider the geometry-based model. With the GB-model, the $(m-1)$-vector $\Delta \tilde{\rho}_{r}$ of $d \tilde{t}_{r}=d \tilde{t}-$ $\Delta \tilde{\rho}_{r}, r=2, \ldots, n$, is parametrized in the $\nu$-vector $\Delta \tilde{x}_{r}$. Hence, for the $k$-epoch case, the $k(m-1) n$ clock parameters of $d \tilde{t}_{r}$ get replaced by the $k(m-1)$ parameters of $d \tilde{t}$ and the $\nu(n-1)$ parameters of $\Delta \tilde{x}_{r}$. The multi-epoch redundancy, for both the ambiguity-float and ambiguityfixed case, increases therefore by $[k(m-1)-\nu][n-1]$ when switching from the GF-model to the GB-model (cf. Table 2).

This increase in model strength will show up in the improved precision of the estimated PPP-RTK corrections. To show this clearly, we have determined the variance matrices of their least-squares GB- and GFestimators under some simplifying assumptions. We followed Teunissen (1997a) and used a time-averaged receiver-satellite geometry matrix $G$ of order $m \times \nu$, in $\tilde{G}=\left[G, e_{m}\right]$, to capture the geometry for the GBmodel ( $e_{m}$ is the $m$-vector of ones). The results are given in Tables 3, 4 and 5, respectively. The proofs of these results can be found in the Appendix.

Table 3 presents the variance matrices of the ambiguity-float GB/GF least-squares PPP-RTK corrections. It shows that all (co)variance matrices, except those of the satellite clocks, follow the 1 -over- $k$ rule. For not too large $k$, however, the same rule applies approximately to the variance matrices of the satellite clocks as well, i.e. $Q_{d \tilde{t} d \hat{\tilde{t}}}^{G B}[k] \approx \frac{1}{k} Q_{d \hat{t} d \hat{\tilde{t}}}^{G B}$ and $Q_{d \hat{t} d \hat{\tilde{t}}}^{G F}[k] \approx \frac{1}{k} Q_{d \hat{\tilde{t}} d \hat{\tilde{t}}}^{G F}$, since $c_{\tilde{\rho}}^{2} \approx 0$.

In the absence of satellite redundancy, we have $m=$ $\nu+1$ and therefore $\tilde{C}_{s}=D_{m}^{T} \mathcal{P} \frac{\perp}{\tilde{G}} C_{s} D_{m}=0$. In that case the ambiguity variance matrix and the ambiguity-clock covariance matrix of the two models are the same. For the variance matrix of the clocks there is then still a slight difference between the GB- and GF-model, one that can be explained by the assumed time-invariance of $\Delta \tilde{x}_{r}$. Thus in the absence of satellite redundancy, the GF-model has approximately the same performance as the GB-model.

Table 4 is the ambiguity-fixed counterpart of Table 3. Again it shows that all (co)variance matrices, except those of the satellite clocks, follow the 1-over- $k$ rule. For not too large $k$, however, the same rule applies approximately to the ambiguity-fixed variance matrices of the satellite clocks as well, i.e. $Q_{d \tilde{t} d \tilde{t}}^{G B}[k] \approx \frac{1}{k} Q_{d \tilde{t} d \check{t}}^{G B}$ and $Q_{d \tilde{t} d \check{\tilde{t}}}^{G F]} \approx k \frac{1}{k} Q_{d \tilde{t} d \check{t}}^{G F}$, since $c_{\check{\rho}}^{2} \approx 0$. Importantly, we note that

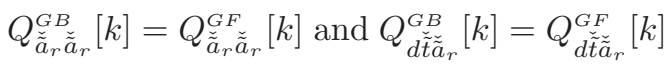

with

$Q_{d \tilde{\tilde{t}} d \tilde{t}}^{G B}[k] \approx Q_{d \tilde{t} d \check{\tilde{t}}}^{G F}[k]$

for not too large $k$. Thus after successful integer ambiguity resolution, the GF-based corrections have a quality that is close to their GB-counterparts.

Let us now consider the impact of integer ambiguity resolution per model. In Table 5 the ambiguity-fixed variance matrices of both models are expressed in their ambiguity-float counterparts. The results show that the 
Table 3 Variance matrices of the $k$-epoch ambiguity-float GB/GF least-squares PPP-RTK corrections expressed in their single-epoch counterparts.

\begin{tabular}{|c|c|c|c|c|}
\hline Multi-epoch GB & & Single-epoch GB & & Multi-epoch GF \\
\hline$Q_{d \hat{\tilde{t}} d \hat{\tilde{t}}}^{G B}[k]$ & $=$ & $\frac{1}{k} Q_{d \hat{\tilde{t}} d \hat{\tilde{t}}}^{G B}[1]+\frac{k-1}{k} \frac{1}{n} c_{\tilde{\rho}}^{2} C_{s}$ & $=$ & $Q_{d \hat{\tilde{t}} d \hat{\tilde{t}}}^{G F}[k]-\frac{1}{k} \frac{n-1}{n} c_{\hat{\rho}}^{2} \tilde{C}_{s}-\frac{k-1}{k} \frac{n-1}{n} c_{\tilde{\rho}}^{2} C_{s}$ \\
\hline$Q_{d \hat{\tilde{t}} \tilde{\tilde{a}}_{1}}^{G B}[k]$ & $=$ & $\frac{1}{k} Q_{d \hat{\tilde{t}} \tilde{\tilde{a}}_{1}}^{G B}[1]$ & $=$ & $Q_{d \tilde{\tilde{t}} \tilde{\tilde{a}}_{1}}^{G F}[k]-\frac{1}{k} \frac{n-1}{n} c_{\hat{\rho}}^{2} e_{\mu}^{T} \Lambda^{-1} \otimes \tilde{C}_{s}$ \\
\hline$Q_{\tilde{\tilde{a}}_{r}}^{G B} \underset{\tilde{\tilde{a}}_{r}}{B}[k]$ & $=$ & $\frac{1}{k} Q_{\tilde{\tilde{a}}_{r}}^{G B} \underset{\tilde{\tilde{a}}_{r}}{B}[1]$ & $=$ & $Q_{\tilde{\tilde{a}}_{r}}^{G F}[k]-\frac{1}{k} \frac{n-1}{n} c_{\hat{\rho}}^{2} \Lambda^{-1} e_{\mu} e_{\mu}^{T} \Lambda^{-1} \otimes \tilde{C}_{s}$ \\
\hline
\end{tabular}

Table 4 Variance matrices of the $k$-epoch ambiguity-fixed GB/GF least-squares PPP-RTK corrections expressed in their single-epoch counterparts.

\begin{tabular}{|c|c|c|c|c|}
\hline Multi-epoch GB & & Single-epoch GB & & Multi-epoch GF \\
\hline$Q_{d \tilde{\tilde{t}} d \tilde{\tilde{t}}}^{G B}[k]$ & $=$ & $\frac{1}{k} Q_{d \tilde{\tilde{t}} d \tilde{\tilde{t}}}^{G B}[1]+\frac{k-1}{k} \frac{1}{n} c_{\tilde{\rho}}^{2} C_{s}$ & $=$ & $Q_{d \tilde{\tilde{t}} d \tilde{t}}^{G F}[k]-\frac{1}{k} \frac{n-1}{n} c_{\tilde{\rho}}^{2} \tilde{C}_{s}-\frac{k-1}{k} \frac{n-1}{n} c_{\tilde{\rho}}^{2} C_{s}$ \\
\hline$Q_{d \tilde{\tilde{t}} \tilde{\tilde{a}}_{1}}^{G B}[k]$ & $=$ & $\frac{1}{k} Q_{d \tilde{\tilde{t}} \tilde{\tilde{a}}_{1}}^{G B}[1]$ & $=$ & $Q_{d \tilde{\tilde{t} \tilde{a}} \tilde{\tilde{a}}_{1}}^{G F}[k]$ \\
\hline$Q_{\tilde{\tilde{a}}_{r} \tilde{\tilde{a}}_{r}}^{G B}[k]$ & $=$ & $\frac{1}{k} Q_{\tilde{\tilde{a}}_{r} \tilde{\tilde{a}}_{r}}^{G B}[1]$ & $=$ & 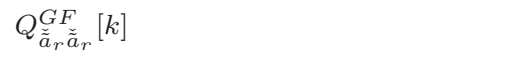 \\
\hline
\end{tabular}

GF (co)variance matrices, except those of the satellite clocks, follow the 1 -over- $n$ rule. For not too large $n$, however, the same rule applies approximately to the GF variance matrix of the satellite clocks as well, i.e. $Q_{d \tilde{\tilde{t}} d \tilde{t}}^{G F}[k] \approx \frac{1}{n} Q_{d \tilde{\tilde{t}} d \hat{\tilde{t}}}^{G F}[k]$, since $c_{\check{\rho}}^{2} \approx 0$.

In case of the GB-model, the impact of ambiguity resolution differs from that of the GF-model. Here the impact largely depends on the strength of the GBmodel. The weaker the model, the larger the impact of ambiguity resolution is. The model is weakest when there is no satellite redundancy. Then $m=\nu+1$ and $\tilde{C}_{s}=0$. At the other extreme we have the geometryfixed case (i.e., $\Delta \tilde{x}_{r}=0$ for all $r$ ). Then $\tilde{C}_{s}=C_{s}$ and no improvement, apart from $Q_{\tilde{\tilde{a}}_{r} \tilde{\tilde{a}}_{r}}^{G B}[k]$, can be realized. In this case, we have with (82) and (83),

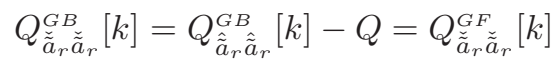

$$
\begin{aligned}
& Q_{d \tilde{t} \tilde{a}_{r}}^{G B}[k]=Q_{d \dot{\tilde{t}} \tilde{\hat{a}}_{r}}^{G B}[k]=Q_{d \tilde{\tilde{t}} \tilde{\tilde{a}}_{r}}^{G F}[k] \\
& Q_{d \tilde{\tilde{t}} d \tilde{t}}^{G B}[k]=Q_{d \tilde{t}}^{G B}\left[\hat{\tilde{t}}[k] \approx Q_{d \tilde{t} d \check{\tilde{t}}}^{G F}[k]\right.
\end{aligned}
$$

with $Q=\frac{n-1}{k n} \Lambda^{-1}\left(C_{\phi}+c_{\hat{\imath} \mid \rho}^{2} \mu \mu^{T}\right) \Lambda^{-1} \otimes \tilde{C}_{s}$.

Hence, the ambiguity-float GB clock solution would then already be as good as that of the ambiguity-fixed solutions of either the GB- or GF-model.

\subsection{Precision of User-Corrected Observations}

Having the variance matrices of the individual PPPRTK corrections available, one can now also determine the variance matrix of the complete user-corrections. In (Odijk et al, 2014b) such example is given for the single-frequency PPP-RTK case. Here we consider as example the dual-frequency GF-model determined corrections. The float corrections that are applied to the user observations are then given as

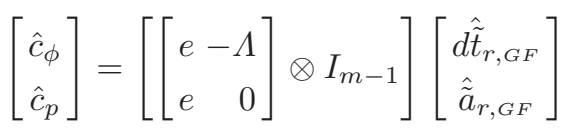

with a likewise definition in case integer ambiguity resolved corrections $d \tilde{\tilde{t}}_{r, G F}$ and $\check{\tilde{a}}_{r, G F}$ are used. The following Lemma gives the variance matrix of these usercorrections for both the ambiguity-float and ambiguityfixed case.

Lemma 4 (Precision GF User-Corrections) The variance matrices of the ambiguity-fixed and ambiguity-float PPP-RTK user-corrections are given for a single-epoch as

$\mathrm{D}\left[\begin{array}{c}\check{c}_{\phi} \\ \check{c}_{p}\end{array}\right]=\frac{1}{n} \mathrm{D}\left[\begin{array}{l}\hat{c}_{\phi} \\ \hat{c}_{p}\end{array}\right]+\frac{n-1}{n} c_{\check{\rho}}^{2}\left[\begin{array}{l}e \\ e\end{array}\right]\left[\begin{array}{l}e \\ e\end{array}\right]^{T} \otimes C_{s}$ 
Table 5 Impact of integer ambiguity resolution: variance matrices of the ambiguity-fixed GB/GF least-squares PPP-RTK corrections expressed in their ambiguity-float counterparts.

\begin{tabular}{|c|c|}
\hline Geometry-based & Geometry-free \\
\hline$Q_{d \tilde{t} d \tilde{t}}^{G B}[k]=Q_{d \tilde{t} d \hat{t}}^{G B}[k]-\frac{1}{k} \frac{n-1}{n}\left(c_{\hat{\rho}}^{2}-c_{\tilde{\rho}}^{2}\right)\left(C_{s}-\tilde{C}_{s}\right)$ & $Q_{d \tilde{t}_{r} d \tilde{t}_{r}}^{G F}[k]=\frac{1}{n} Q_{d \tilde{t}_{r} d \hat{t}_{r}}^{G F}[k]+\frac{n-1}{n} c_{\tilde{\rho}}^{2} C_{s}$ \\
\hline$Q_{d \tilde{t} \tilde{a}_{1}}^{G B}[k]=Q_{d \tilde{\hat{t}} \hat{\tilde{a}}_{1}}^{G B}[k]-\frac{1}{k} \frac{n-1}{n} c_{\hat{\rho}}^{2} e_{\mu}^{T} \Lambda^{-1} \otimes\left(C_{s}-\tilde{C}_{s}\right)$ & $Q_{d \tilde{t}_{r} \tilde{a}_{r}}^{G F}[k]=\frac{1}{n} Q_{d \hat{t}_{r} \hat{a}_{r}}^{G F}[k]$ \\
\hline$Q_{\tilde{\tilde{a}}_{r} \tilde{\tilde{a}}_{r}}^{G B}[k]=Q_{\hat{\tilde{a}}_{r} \hat{\tilde{a}}_{r}}^{G B}[k]-\frac{1}{k} \frac{n-1}{n} c_{\hat{\rho}}^{2} \Lambda^{-1} e_{\mu} e_{\mu}^{T} \Lambda^{-1} \otimes\left(C_{s}-\tilde{C}_{s}\right)-\frac{1}{k} \frac{n-1}{n} \Lambda^{-1}\left(C_{\phi}+c_{\hat{\imath} \mid \rho}^{2} \mu \mu^{T}\right) \Lambda^{-1} \otimes \tilde{C}_{s}$ & $Q_{\tilde{a}_{r}}^{G F} \tilde{\tilde{a}}_{r}[k]=\frac{1}{n} Q_{\hat{\tilde{a}}_{a}}^{G F \hat{a}_{r}}[k]$ \\
\hline
\end{tabular}

and

$$
\begin{aligned}
\mathrm{D}\left[\begin{array}{c}
\hat{c}_{\phi} \\
\hat{c}_{p}
\end{array}\right]= & \left(\left[\begin{array}{cc}
C_{\phi} & 0 \\
0 & C_{p}
\end{array}\right]+c_{\hat{\imath}}^{2}\left[\begin{array}{c}
-\mu \\
\mu
\end{array}\right]\left[\begin{array}{c}
-\mu \\
\mu
\end{array}\right]^{T}+\right. \\
& \left.-\left[\begin{array}{c}
-\mu \\
\mu
\end{array}\right]\left[\begin{array}{c}
0 \\
C_{p} \mu_{G F}
\end{array}\right]^{T}-\left[\begin{array}{c}
0 \\
C_{p} \mu_{G F}
\end{array}\right]\left[\begin{array}{c}
-\mu \\
\mu
\end{array}\right]^{T}\right) \otimes C_{s}
\end{aligned}
$$

with $c_{\hat{\iota}}^{2}=\mu_{G F}^{T} C_{p} \mu_{G F}$.

Proof Follows from using the relevant entries of Tables 3 and 4 in the application of the variance propagation law to $(85)$.

Two important remarks can now be made about these variance matrices. First, since these variance matrices describe the precision of the user-corrections, all their entries are needed when one wants to perform a statistical validation of the user-corrections themselves (either ambiguity-fixed or ambiguity-float based). For the user-processing however, not all entries are needed. To understand this, consider the sum-structure of the variance matrices (86) and (87). In them one will recognize rank-one matrices with components that lie in the range-space of the design matrix of the user-system of observation equations. These are the rank-one matrices with components $\left[e^{T}, e^{T}\right]^{T}$ or $\left[-\mu^{T}, \mu^{T}\right]^{T}$ (three in 87 and four in 86). Since they lie in the range-space of the user's design matrix, this part of the variance matrix of the user-corrections will not contribute to the estimation of the user parameters. Hence, for the userprocessing only the following components are of relevance,

$\frac{1}{n}\left[\begin{array}{ll}C_{\phi} & 0 \\ 0 & C_{p}\end{array}\right] \otimes C_{s}$ or $\left[\begin{array}{ll}C_{\phi} & 0 \\ 0 & C_{p}\end{array}\right] \otimes C_{s}$

depending on whether the ambiguity-fixed or ambiguityfloat corrections are used. Note that this result is consistent with our earlier relative positioning conclusion of PPP-RTK (cf. section 4.3). It thus implies that the $\left[e^{T}, e^{T}\right]^{T}$ and $\left[-\mu^{T}, \mu^{T}\right]^{T}$ induced correlation present in (86) and (87) can be neglected.

In the first matrix of (88) we see the contribution of the number of network stations, which is analogous to the contribution in the array-aided PPP concept of Teunissen (2012). An increasing number of stations will thus in particular help to reduce the noise contribution of the code measurements.

\subsection{Integer Ambiguity Resolution}

\subsubsection{ILS, IB and IR}

The ambiguity resolved estimators of the previous subsections can only be obtained once integer ambiguity resolution has been performed. We now show how the integer estimators of $\tilde{z}_{i}, i=2, \ldots, n,($ cf. 78 ) can be obtained.

Defining the ambiguity matrices $\mathcal{A}=\left[\tilde{a}_{1}, \ldots, \tilde{a}_{n}\right]$, $\mathcal{Z}=\left[\tilde{z}_{2}, \ldots, \tilde{z}_{n}\right]$, and using the property $\tilde{a}_{r}=\tilde{z}_{r}-\tilde{\delta}$, with $\tilde{z}_{r} \in \mathbb{Z}^{2(m-1)}$ (cf. 23), we can write

$\mathcal{Z}=\mathcal{A} D_{n} \in \mathbb{Z}^{2(m-1) \times(n-1)}$

with $D_{n}=\left[-e_{n-1}, I_{n-1}\right]^{T}$ the between-station differencing matrix. The Integer Least-Squares (ILS) estimator of $\mathcal{Z}$ can then be computed as

$\check{\mathcal{Z}}=\arg \min _{Z \in \mathbb{Z}^{2}(m-1) \times(n-1)}\left\|\operatorname{vec}\left(\hat{\mathcal{A}} D_{n}-Z\right)\right\|_{\mathrm{D}\left(\operatorname{vec}\left(\hat{\mathcal{A}} D_{n}\right)\right)}^{2}$

with $\mathrm{D}\left(\operatorname{vec}\left(\hat{\mathcal{A}} D_{n}\right)\right)$ the variance matrix of the float solution $\operatorname{vec}\left(\hat{\mathcal{A}} D_{n}\right)$, obtained from either the GF or the GB model. The single-epoch version of this variance matrix is given as

$\mathrm{D}\left(\operatorname{vec}\left(\hat{\mathcal{A}} D_{n}\right)\right)=D_{n}^{T} D_{n} \otimes C_{a a} \otimes D_{m}^{T} C_{S} D_{m}$

with

$C_{a a}=\left\{\begin{array}{l}C_{a a}^{\mathrm{GFi}}=\Lambda^{-1}\left(C_{\phi}+c_{\hat{\imath} \mid \rho}^{2} \mu \mu^{T}\right) \Lambda^{-1} \\ C_{a a}^{\mathrm{GF}}=C_{a a}^{\mathrm{GFi}}+\Lambda^{-1}\left(c_{\hat{\rho}}^{2} e_{\mu} e_{\mu}^{T}\right) \Lambda^{-1}\end{array}\right.$

in which $C_{a a}^{\mathrm{GFi}}$ and $C_{a a}^{\mathrm{GF}}$ denote the geometry-fixed and geometry-free ambiguity cofactor matrices, respectively. The geometry-fixed and geometry-free cases are considered, since they represent the two extreme cases of model strength.

The ILS estimator (90) is optimal in the sense that it has the largest possible success-rate of all integer estimators (Teunissen, 1999). Popular alternatives to 
ILS are integer bootstrapping (IB) and integer rounding (IR). They are easier to compute than ILS and when the model has sufficient strength their performance can be close to optimal once the decorrelating Z-transformation is applied (Teunissen, 1995, 1998).

One can also approximate the ILS solution (90) by approximating the variance matrix. The objective function of (90) decouples, for instance, and becomes easier to minimize, if one neglects the correlation due to differencing. Would one neglect the correlation all together and replace $\mathrm{D}\left(\operatorname{vec}\left(\hat{\mathcal{A}} D_{n}\right)\right)$ in $(90)$ by a diagonal matrix, then the solution would reduce to ordinary componentwise integer rounding,

$\check{\tilde{z}}_{i}=\left\lfloor\hat{\tilde{a}}_{i}-\hat{\tilde{a}}_{1}\right\rceil, i=2, \ldots, n$

If the underlying model is too weak to achieve high enough success-rates for full ambiguity resolution, one can also opt for partial ambiguity resolution. Resolving only wide-lane ambiguities, for instance, is a special case of partial ambiguity resolution (Teunissen, 1997d).

\subsubsection{Ambiguity Dilution of Precision}

To get an indication of the ambiguity resolution strength of the models, we use the Ambiguity Dilution of Precision (ADOP). This is an easy-to-apply diagnostic measure that was introduced by Teunissen (1997b). The $\mathrm{ADOP}$ is defined as the square-root of the ambiguity variance matrix' determinant taken to the power one over the matrix' order. Thus in case of full-ambiguity resolution, with $m$ satellites, $n$ stations and 2 frequencies, it is defined as

$\mathrm{ADOP}=\left|\mathrm{D}\left(\operatorname{vec}\left(\hat{\mathcal{A}} D_{n}\right)\right)\right|^{\frac{1}{4(m-1)(n-1)}} \quad($ cycle $)$

Since the ADOP is a measure for the average ambiguity precision, the ADOP can also be linked to the probability of correct integer estimation, the ambiguity successrate. As a rule of thumb, ADOP-values smaller than about 0.10 cycle, correspond to ADOP-based successrates larger than 0.999 (Odijk and Teunissen, 2008).

If the ADOP-values for full ambiguity resolution are too large, one may also consider partial ambiguity resolution. If we denote the wide-lane-only ambiguity dilution of precision as $\mathrm{ADOP}_{W L}$, then it follows from the definition of the ambiguity dilution of precision that for the dual-frequency case,

$\mathrm{ADOP}^{2}=\mathrm{ADOP}_{L_{1} \mid W L} \times \operatorname{ADOP}_{W L} \quad\left(\operatorname{cycle}^{2}\right)$

with $\mathrm{ADOP}_{L_{1} \mid W L}$ denoting the wide-lane conditioned $L_{1}$-only ambiguity dilution of precision. Thus if two of the entries in (95) are given, the third follows.

The following lemma gives analytical expressions for the geometry-fixed and geometry-free ADOPs in case of full ambiguity resolution and wide-lane-only ambiguity resolution.

Lemma 5 (Geometry-fixed and Geometry-free ADOPs) Assuming $C_{\phi}=\sigma_{\phi}^{2} I_{2}$ and $C_{p}=\sigma_{p}^{2} I_{2}$ (cf. 75), the single epoch, geometry-fixed (GFi) and geometry-free (GF) ADOPs of full-ambiguity resolution and wide-lane-only ambiguity resolution are given as

$$
\begin{aligned}
\mathrm{ADOP}^{\mathrm{GFi}} & =c_{o}\left(\frac{\sigma_{\phi} \sigma_{p}}{\lambda_{1} \lambda_{2}}\right)^{\frac{1}{2}}(1+\epsilon)^{\frac{1}{4}} \\
\mathrm{ADOP}^{\mathrm{GF}} & =\mathrm{ADOP}^{\mathrm{GFi}}\left(\frac{1+\epsilon}{\epsilon}+\frac{4\left(\mu_{1}+\mu_{2}\right)^{2}}{(1+\epsilon)\left(\mu_{2}-\mu_{1}\right)^{2}}\right)^{\frac{1}{4}}
\end{aligned}
$$

and

$\operatorname{ADOP}_{W L}^{\mathrm{GFi}}=c_{O}\left(\left(\frac{\sigma_{\phi}^{2}}{\lambda_{1}^{2}}+\frac{\sigma_{\phi}^{2}}{\lambda_{2}^{2}}\right)+\frac{\sigma_{p}^{2}}{\lambda_{W}^{2}} \frac{\mu_{2}}{\mu_{1}^{2}+\mu_{2}^{2}}\right)^{\frac{1}{2}}$

$\operatorname{ADOP}_{W L}^{\mathrm{GF}}=\operatorname{ADOP}_{W L}^{\mathrm{GFi}}(1+\gamma)^{\frac{1}{2}}$

with $\epsilon=\sigma_{\phi}^{2} / \sigma_{p}^{2}$ and

$c_{o}=n^{\frac{1}{2(n-1)}}\left(\sum_{s=1}^{m} c_{s}^{-2} / \prod_{s=1}^{m} c_{s}^{-2}\right)^{\frac{1}{2(m-1)}}$

where $c_{s}^{2}$ are the diagonal entries of the elevation-weighting matrix $C_{S}$. The scalar $\gamma$ is approximated as $\gamma \approx\left(f_{1}^{3}-\right.$ $\left.f_{2}^{3}\right)^{2} /\left(f_{1}^{2} f_{2}^{2}\left(f_{1}+f_{2}\right)^{2}\right)$.

Proof Follows from an application of the results of Odijk and Teunissen (2008). The exact value of $\gamma$ is given in Appendix.

The Lemma shows that for full ambiguity resolution, the geometry-free ADOP (cf. 96) is about a factor $\left(\frac{1+\epsilon}{\epsilon}\right)^{\frac{1}{4}} \approx$ $10\left(\epsilon \approx 10^{-4}\right)$ larger than the corresponding geometryfixed ADOP. The Lemma also shows that while the geometry-fixed wide-lane-only ADOP is governed by $\sigma_{p} / \lambda_{W}$ (cf. 97), its full-ambiguity resolution counterpart is governed by $\left(\sigma_{\phi} \sigma_{p} / \lambda_{1} \lambda_{2}\right)^{\frac{1}{2}}$. Hence, for the geometry-fixed case it does not pay off to do wide-lane-only ambiguity resolution. This changes if one considers the geometry-free model. In that case, wide-lane-only ambiguity resolution has a better success-rate performance, since the full-ambiguity resolution geometry-free ADOP is about a factor 6 larger than its wide-lane-only counterpart, while the wide-lane-only geometry-free ADOP does not differ too much from its geometry-fixed counterpart.

Numerical values for the ADOPs are given in Table 6 . They show that full ambiguity resolution requires many epochs in case of the geometry-free model, but only in the order of about ten for the geometry-fixed model. In contrast to full ambiguity resolution, the table also shows that wide-lane-only ambiguity resolution is possible with the geometry-free model. And once the wide-lane ambiguities are known, the geometry-fixed model allows for a reasonable quick resolution of the $L_{1}$ ambiguities. This is in contrast to the geometry-free model. 
Table 6 Single-epoch, zenith-referenced ADOP-values for the geometry-fixed and geometry-free scenarios (GPS dual-frequency L1/L2). ADOP refers to full ambiguity resolution; $\mathrm{ADOP}_{W L}$ to wide-lane-only ambiguity resolution, while $\mathrm{ADOP}_{L_{1} \mid W L}$ refers to $L_{1}$ ambiguity resolution assuming the wide-lane ambiguities known.

\begin{tabular}{lcc}
\hline \multicolumn{1}{c}{$\mathrm{ADOP}[$ cycle] } & Geometry-fixed & Geometry-free \\
\hline $\mathrm{ADOP}$ & 0.278 & 2.787 \\
$\mathrm{ADOP}_{W L}$ & 0.465 & 0.497 \\
$\operatorname{ADOP}_{L_{1} \mid W L}$ & 0.166 & 15.620 \\
\hline$m=2 ; n=2 ; \sigma_{\phi}=3[\mathrm{~mm}] ; \sigma_{p}=30[\mathrm{~cm}]$
\end{tabular}

\section{The UPD/FCB method revisited}

The estimation methods and results presented in the previous sections apply to all the discussed PPP-RTK models. As all the PPP-RTK models considered above are shown to be intrinsically equivalent, a rigorous application of these estimation methods to the different models will therefore result in identical positioning results. This is also true for the model underlying the UPD/FCB method. However, as the UPD/FCB estimation method itself differs from the methods of estimation of the previous sections, see e.g. (Ge et al, 2008; Geng et al, 2010, 2011, 2012; Geng and Bock, 2013), it is of interest to identify and understand what those differences are.

\subsection{The UPD/FCB estimation method}

Here we follow (Ge et al, 2008, p. 392-393) and (Geng et al, 2012, p. 580-582). In the UPD/FCB estimation method, the sequence of estimating the corrections $d \tilde{t}^{p s}$, $\operatorname{frac}\left(\tilde{a}_{r, c}^{p s}\right)$ and $\operatorname{frac}\left(\tilde{a}_{r, W}^{p s}\right)$ (cf. 54) is as follows. First, a geometry-free approach is used to obtain an estimate of the fractional part of the wide-lane ambiguity $\tilde{a}_{r, W}^{p s}$. Then, a geometry-based approach is used to obtain a float estimate of the satellite clock $d \tilde{t}^{p s}$. Finally, a geometry-free approach is used again to obtain an estimate of the fractional part of $\tilde{a}_{r, c}^{p s}$. More specifically the three steps can be described as follows:

Step 1: The computation of the fractional wide-lane estimate is based on the third equation of the FCB network-model (43 or 53):

$\Delta \phi_{r, W N}^{p s}=\lambda_{W} \tilde{a}_{r, W}^{p s}, r=1, \ldots, n$

This system is solved on a station-by-station basis, the solutions of which are time-averaged to give the estimates $\hat{\tilde{a}}_{r, W}^{p s}, r=1, \ldots, n$. The fractional parts of them are then station averaged to give (Geng and Bock, 2013, p. 451, Eq. (4)),

$\overline{\operatorname{frac}}\left(-\tilde{\delta}_{, W}^{p s}\right)=\frac{1}{n} \sum_{r=1}^{n} \operatorname{frac}\left(\hat{\tilde{a}}_{r, W}^{p s}\right)$

Step 2: The computation of the estimate for $d \tilde{t}^{p s}$ is based on the first two equations of (53),

$$
\begin{aligned}
\Delta \phi_{r, I F}^{p s}-\frac{\lambda_{2}}{\mu_{12}}\left\lfloor\tilde{a}_{r, W}^{p s}\right\rceil & =\Delta \tilde{\rho}_{r}^{p s}-d \tilde{t}^{p s}+\lambda_{N} \tilde{a}_{r, c}^{p s} \\
\Delta p_{r, I F}^{p s} & =\Delta \tilde{\rho}_{r}^{p s}-d \tilde{t}^{p s}, r=1, \ldots, n
\end{aligned}
$$

For $\left\lfloor\tilde{a}_{r, W}^{p s}\right\rceil$ the integer $\left\lfloor\hat{\tilde{a}}_{r, W}^{p s}\right\rceil$ based on $\hat{\tilde{a}}_{r, W}^{p s}$ of the previous step is used. The system is solved to obtain $\Delta \hat{\tilde{\rho}}_{r}$, $\hat{\tilde{t}}^{p s}$ and $\hat{\tilde{a}}_{r, c}^{p s}$ (Geng et al, 2010, p. 582). Note that this step is not necessary if we have a precise satellite clock product.

Step 3: In this last step the fractional parts of the 'narrow-lane' ambiguity solutions $\hat{\tilde{a}}_{r, c}^{p s}, r=1, \ldots, n$, of the previous step are taken to compute the station averaged estimate (Ge et al, 2008; Geng et al, 2012, p. 582, Eq. (11))

$\overline{\operatorname{frac}}\left(-\tilde{\delta}_{, c}^{p s}\right)=\frac{1}{n} \sum_{r=1}^{n} \operatorname{frac}\left(\hat{\tilde{a}}_{r, c}^{p s}\right)$

Hence, as a result of the above three steps, the PPPRTK corrections provided are the clocks estimate $d \hat{\tilde{t}}^{p s}$ and the two FCB estimates $\overline{\operatorname{frac}}\left(-\tilde{\delta}_{, c}^{p s}\right)$ and $\overline{\operatorname{frac}}\left(-\tilde{\delta}_{, W}^{p s}\right)$, respectively. In the improved narrow-lane FCB method of Geng et al (2012, Sect. 2.3), the computation (102) is replaced by its ambiguity resolved counterpart, through which an improvement in the results, over the method proposed by Ge et al (2008), was reported.

The above estimators (100) and (102) relate to between-satellite phase biases. Would one work with undifferenced observations, similar estimators can be formulated for the fractional part of the between-receiver phase biases, see e.g., (Li et al, 2013b, 2014).

\subsection{Comparison of Estimators}

We now compare the FCB-estimators (100) and (102) with their corresponding ambiguity resolved satellite phase bias estimator. We start with the wide-lane version (100). Since $\tilde{a}_{r}^{p s}=\tilde{z}_{r}^{p s}-\tilde{\delta}^{p s}$ (cf. 23) and thus $\tilde{a}_{r, W}^{p s}=\tilde{z}_{r, W}^{p s}-\tilde{\delta}_{, W}^{p s}$, we have, since $\tilde{z}_{r, W}^{p s} \in \mathbb{Z}$, that $\operatorname{frac}\left(\tilde{a}_{r, W}^{p s}\right)=\operatorname{frac}\left(-\tilde{\delta}_{, W}^{p s}\right)$ for $r=1, \ldots, n$, and therefore

$\operatorname{frac}\left(-\tilde{\delta}_{, W}^{p s}\right)=\frac{1}{n} \sum_{r=1}^{n} \operatorname{frac}\left(\tilde{a}_{r, W}^{p s}\right)$ 
Having station-estimators $\hat{\tilde{a}}_{r, W}^{p s}$ of $\tilde{a}_{r, W}^{p s}$ available $(r=$ $1, \ldots, n)$, it is then perhaps 'natural', as is done in the FCB estimation method, to take as estimator of the fraction $\operatorname{frac}\left(-\tilde{\delta}_{, W}^{p s}\right)$, the station-average (100),

$\overline{\operatorname{frac}}\left(-\tilde{\delta}_{, W}^{p s}\right)=\frac{1}{n} \sum_{r=1}^{n} \operatorname{frac}\left(\hat{\tilde{a}}_{r, W}^{p s}\right)$

We now compare this 'natural' estimator with the best estimator of the wide-lane phase-bias $\tilde{\delta}_{W}^{p s}$, i.e. the ambiguity resolved estimator $\check{\tilde{\delta}}_{, W}^{p s}$. Recall that the integer estimate $\check{\widetilde{z}}_{j}^{p s}$ in (80) follows from applying integer ambiguity resolution to the GF float solution $\hat{\tilde{z}}_{j}=$ $\hat{\tilde{a}}_{j, G F}-\hat{\tilde{a}}_{1, G F}$. Hence, if integer rounding is used on its wide-lane component to obtain the integer estimate $\check{\tilde{z}}_{j, W}^{p s}=\left\lfloor\hat{\tilde{z}}_{j, W}^{p s}\right\rceil=\left\lfloor\hat{\tilde{a}}_{j, W}^{p s}-\hat{\tilde{a}}_{1, W}^{p s}\right\rceil$, it follows from $(80)$ that

$$
\begin{aligned}
\check{\tilde{\tilde{\delta}}}_{, W}^{p s} & =-\frac{1}{n} \sum_{j=1}^{n}\left(\hat{\tilde{a}}_{j, W}^{p s}-\left\lfloor\hat{\tilde{a}}_{j, W}^{p s}-\hat{\tilde{a}}_{1, W}^{p s}\right\rceil\right) \\
& =-\frac{1}{n} \sum_{j=1}^{n}\left(\left(\hat{\tilde{a}}_{j, W}^{p s}-\hat{\tilde{a}}_{1, W}^{p s}\right)-\left\lfloor\hat{\tilde{a}}_{j, W}^{p s}-\hat{\tilde{a}}_{1, W}^{p s}\right\rceil\right)-\hat{\tilde{a}}_{1, W}^{p s} \\
& =-\frac{1}{n} \sum_{j=1}^{n} \operatorname{frac}\left(\hat{\tilde{a}}_{j, W}^{p s}-\hat{\tilde{a}}_{1, W}^{p s}\right)-\hat{\tilde{a}}_{1, W}^{p s} \\
& =-\frac{1}{n} \sum_{j=1}^{n} \operatorname{frac}\left(\hat{\tilde{z}}_{j, W}^{p s}\right)-\hat{\tilde{a}}_{1, W}^{p s}
\end{aligned}
$$

and therefore

$-\check{\tilde{\delta}}_{, W}^{p s}=\frac{1}{n} \sum_{j=1}^{n} \operatorname{frac}\left(\hat{\tilde{z}}_{j, W}^{p s}\right)+\hat{\tilde{a}}_{1, W}^{p s}$

This ambiguity resolved estimator of the wide-lane phasebias is made up of two different terms. A station-average of the fractions of double-differenced float wide-lane ambiguities and the single-differenced float wide-lane ambiguity of the reference station 1 . Thus although this estimator is also made up from a station average, its fraction is quite different from the FCB-estimator (104). We therefore have the following result.

Lemma 6 (FCB-estimator) The station-averaged FCB estimator $\frac{1}{n} \sum_{r=1}^{n} \operatorname{frac}\left(\hat{\tilde{a}}_{r, W}^{p s}\right)$ (cf. 100) is not optimal, since

$\overline{\operatorname{frac}}\left(\tilde{\delta}_{, W}^{p s}\right) \neq \operatorname{frac}\left(\check{\tilde{\delta}}_{, W}^{p s}\right)$ for $n>1$

Thus only in case of a single station, $n \underset{\sim \sim p s}{=} 1$, do we have $\operatorname{frac}\left(\check{\tilde{\delta}}_{, W}^{p s}\right)=\overline{\operatorname{frac}}\left(\tilde{\delta}_{, W}^{p s}\right)$, since then $-\check{\tilde{\delta}}_{, W}^{p s}=\tilde{\tilde{a}}_{1, W}^{p s}$, because $\hat{\tilde{z}}_{1, W}^{p s}=0$ by definition. A similar result as (107) also holds true for the estimator (102). Note that in Ge et al. (2008) the estimators $\overline{\operatorname{frac}}\left(\tilde{\delta}_{, W}^{p s}\right)$ and $\overline{\operatorname{frac}}\left(\tilde{\delta}_{, c}^{p s}\right)$ are used, while in Geng et al. (2012) the estimators $\overline{\operatorname{frac}}\left(\tilde{\delta}_{, W}^{p s}\right)$ and improved narrow-lane FCBs are used.

\subsection{Unbiasedness}

Although the FCB-estimator (100) is not optimal, it could still be a useful estimator if it does what it is supposed to do, namely to guarantee that the userambiguities become integer when the estimator is applied as a PPP-RTK correction. Unfortunately, this is not the case either.

This can be understood, if one considers (104) as estimator of (103). Clearly, if $\hat{\tilde{a}}_{r, W}^{p s}$ is an unbiased estimator of $\tilde{a}_{r, W}^{p s}$, then $\overline{\operatorname{frac}}\left(-\tilde{\delta}_{, W}^{p s}\right)$ is not an unbiased estimator of frac $\left(-\tilde{\delta}_{, W}^{p s}\right)$, not even of an integer-shifted version of it. Since the frac-operator is nonlinear, the expectation operator and frac-operator do not commute. We therefore have the following result.

Lemma 7 (FCB-estimator as PPP-RTK correction) The station-averaged PPP-RTK correction $\frac{1}{n} \sum_{r=1}^{n} \operatorname{frac}\left(\hat{\tilde{a}}_{r, W}^{p s}\right)$ (cf. 100) of the FCB estimation method fails to recover the integerness of the user-ambiguities, since

$\mathrm{E}\left(\overline{\operatorname{frac}}\left(\tilde{\delta}_{, W}^{p s}\right)\right) \neq \operatorname{frac}\left(\tilde{\delta}_{, W}^{p s}\right)+z$ for some $z \in \mathbb{Z}$

Using the station-average $\frac{1}{n} \sum_{r=1}^{n} \operatorname{frac}\left(\hat{\tilde{a}}_{r, W}^{p s}\right)$ as estimator for the PPP-RTK correction $\operatorname{frac}\left(\tilde{a}_{q, W}^{p s}\right)=-\operatorname{frac}\left(\tilde{\delta}_{, W}^{p s}\right)$ in (48) or (54), will thus bias the user-ambiguities into noninteger values, and thereby undermine the whole purpose of PPP-RTK. Would one use this FCB-estimation method and resolve the user-ambiguities as if they are integer, one would in fact propagate their non-integerness as bias into the estimators of the remaining parameters. The following simple example makes this clear.

Example: Consider the model of two observation equations with two unknowns,

$\mathrm{E}\left[\begin{array}{c}\phi+a_{i} \\ p\end{array}\right]=\left[\begin{array}{ll}1 & 1 \\ 1 & 0\end{array}\right]\left[\begin{array}{l}\rho \\ z_{i}\end{array}\right]$

with real-valued parameter $\rho$ and integer-valued parameter $z_{i}$. For simplicity, we assume the variance matrix of the observables to be a scaled unit-matrix. The ILS solution of $\rho$ follows then as

$$
\begin{aligned}
\check{\rho}_{i} & =\frac{1}{2}\left(\phi+a_{i}+p-\left\lfloor\phi+a_{i}-p\right\rceil\right) \\
& =\frac{1}{2}\left(\phi+\operatorname{frac}\left(a_{i}\right)+p-\left\lfloor\phi+\operatorname{frac}\left(a_{i}\right)-p\right\rceil\right)
\end{aligned}
$$

The second equation follows from the first, since integer rounding is integer equivariant, i.e. $\lfloor x+z\rceil=\lfloor x\rceil+z$ for $z \in \mathbb{Z}$ and thus $\operatorname{frac}(x+z)=\operatorname{frac}(x)$. Hence, the result (110) shows that the ILS solution $\check{\rho}_{i}$ remains unchanged when $a_{i}$ in (109) is replaced by frac $\left(a_{i}\right)$.

Let us now assume that $i=1, \ldots, n$ and that we replace $a_{i}$ in the first equation of (110) by the average $\frac{1}{n} \sum_{i=1}^{n} \operatorname{frac}\left(a_{i}\right)$. The resulting estimator reads then

$$
\begin{gathered}
\check{\rho}^{\prime}= \\
\frac{1}{2}\left(\phi+\frac{1}{n} \sum_{i=1}^{n} \operatorname{frac}\left(a_{i}\right)+p-\left\lfloor\phi+\frac{1}{n} \sum_{i=1}^{n} \operatorname{frac}\left(a_{i}\right)-p\right\rceil\right)
\end{gathered}
$$


This estimator, however, is a biased estimator of $\rho$. This can be understood as follows. Since the ILS estimators $\check{\rho}_{i}, i=1, \ldots, n$, are unbiased estimators of $\rho$ (Teunissen, 1999), their average

$$
\begin{aligned}
\check{\rho} & =\frac{1}{n} \sum_{i=1}^{n} \check{\rho}_{i} \\
& =\frac{1}{2}\left(\frac{1}{n} \sum_{i=1}^{n}\left(\phi+\operatorname{frac}\left(a_{i}\right)+p-\left\lfloor\phi+\operatorname{frac}\left(a_{i}\right)-p\right\rceil\right)\right)
\end{aligned}
$$

is also unbiased, i.e. $\mathrm{E}(\check{\rho})=\rho$. Hence, the bias in $\check{\rho}^{\prime}$ is given by the expectation of the difference between (111) and (112) as,

$$
\begin{gathered}
\mathrm{E}\left(\check{\rho}^{\prime}-\check{\rho}\right)= \\
\frac{1}{2} \mathrm{E}\left(\frac{1}{n} \sum_{i=1}^{n}\left\lfloor\phi-p+\operatorname{frac}\left(a_{i}\right)\right\rceil-\left\lfloor\phi-p+\frac{1}{n} \sum_{i=1}^{n} \operatorname{frac}\left(a_{i}\right)\right\rceil\right)
\end{gathered}
$$

This bias is nonzero in general. It is zero for $n=1$ and it is zero for the case that all $a_{i}$ 's can be treated as being equal to an integer shifted version of the same constant, say $-\delta$. Thus $a_{i}=z_{i}-\delta$ must hold, instead of $\mathrm{E}\left(a_{i}\right)=z_{i}-\delta$.

\section{Conclusions and Summary}

In this contribution six different dual-frequency PPPRTK models were reviewed and compared: two common clock (CC) models, the distinct clocks (DC) model, the integer recover clock (IRC) model, the decoupled satellite clock (DSC) model and the uncalibrated phase delay/fractional cycle bias (UPD/FCB) model. We discussed both their network-component and user-component. Furthermore, by application of $\mathcal{S}$-system theory, we identified the estimable parameters involved in each of the different methods. The interpretation of these estimable parameters is essential for gaining a proper insight into the principles of PPP-RTK in general, and into the role of the PPP-RTK corrections in particular.

We made a distinction between the model formulation used and the estimation method employed. As to the model formulation, we considered the $\mathcal{S}$-basis choice, the chosen parameterization and the ionospheric delays. From the analyses followed that the CC-1, DC and IRC/DSC models all use the same $\mathcal{S}$-basis, namely $\left(\Delta x_{1}, d^{p s}, z_{1}^{p s}\right)^{T}$, and that the IRC/DSC model is a reparametrized form of both the $\mathrm{DC}$ model and the CC-1 model, using ionosphere-free observations. The IRC/DSC integer recovery or decoupled satellite clock was shown to be the ionosphere-free version of the DC's distinct clock.

All the four models, CC-1, DC and IRC/DSC, have integer ambiguities in their network system of observation equations because of the chosen $\mathcal{S}$-basis. This is not the case with the other two models, the CC-2 model and the (UPD/FCB) model. These two models use $\left(\Delta x_{1}, d^{p s}, \delta^{p s}\right)^{T}$ as $\mathcal{S}$-basis, whereby the (UPD/FCB) model was shown to be a reparametrized form of the ionosphere-free version of the CC-2 model. As we consider the lack of ionospheric information the bottleneck for fast ambiguity resolution, the advantage of working with the original undifferenced formulations over the ionosphere-free formulations was also pointed out.

In the construction of its PPP-RTK corrections, the (UPD/FCB) model makes use of a fractional operator. Although it is not essential, the use of this single operator is permitted as it maintains the integerness of the user-ambiguities. However, as it was pointed out, one has to be aware that, from a probabilistic point of view, an application of the fractional operator changes the statistics of the user-corrected observables. Hence, one has to take this into account when evaluating the statistics and quality of the user-corrected observables. This aspect does not yet seem to be fully developed in the applications that make use of the FCB model.

Although the six models provide for different PPPRTK corrections, an estimability analysis showed their information content to be the same. This implies that they are related through one-to-one transformations. These transformations were given in Table 1. They have the practical implication of showing how the different PPP-RTK methods can be mixed between network and users.

Our estimability analysis also revealed the intrinsic role that is played by the PPP-RTK corrections in establishing the link between user-parameters and network-parameters. It was shown that the single-receiver 'user' integer ambiguities are straightforward doubledifferenced ambiguities and not undifferenced ambiguities as is sometimes stated. Hence, the integer ambiguity resolution at the PPP-RTK user-side is always that of double-differenced ambiguities (or $Z$-transformed functions thereof).

Similarly, it was shown that the PPP-RTK corrections, next to establishing an ambiguity link, also establish a positional link between network and user. Through the corrections, the user-positioning parameters become in essence relative-positioning parameters between user and network. PPP-RTK is thus a relative positioning method and not one of absolute positioning. This was further demonstrated by showing that one recovers the single-baseline model if one considers the extreme case of a PPP-RTK network consisting of a single station only.

As an extension to the review, we presented four different least-squares PPP-RTK estimators. They are the float and fixed estimators under the geometry-free (GF) model and the float and fixed estimators under 
the geometry-based (GB) model. As these estimators are generally applicable, they apply to any of the PPPRTK methods discussed. In order to understand the precision with which the corrections can be estimated, analytical expressions for their variance matrices were derived. This was done for the CC-2 model, having realvalued ambiguities. With the transformations of Table 1 , the corresponding variance matrices of the PPP-RTK corrections for the other models are easily obtained.

Using the analytical expressions of the variance matrices, it was shown that the precision of the ambiguityfixed corrections did not differ too much between the GF- and the GB-model. Thus once ambiguity resolution has been successfully applied, either the GF-based or GB-based corrections can be used.

In the impact of ambiguity resolution on their precision, the GF-based and GB-based corrections do differ however. In case of the GF-model, the varianceimprovement due to ambiguity resolution follows the 1-over- $n$ rule ( $n$ being the number of network stations). In case of the GB-model however, this improvement depends on its model-strength. It is larger, the weaker the model is, and it becomes minimum in the geometryfixed case. In this latter case, the ambiguity-float GB clock solution is already as good as that of the ambiguityfixed solution.

In providing the analytical expressions for the variance matrices of the individual PPP-RTK corrections, we also determined the variance matrix of the complete user-corrections. Here it was shown that in dependence of its use, not all of its entries need to be known. Although the complete matrix is needed for the statistical validation of the corrections themselves, such is not needed for the actual user-processing. For this latter case, it suffices to neglect the variance-covariance components that are related to geometry and ionosphere.

We also analysed the ambiguity resolution strength of the models by means of analytical expressions of their ADOPs. It clearly showed the difference in strength (about a factor of 10) between the geometry-fixed and geometry-free cases. Moreover it showed that in contrast to the geometry-free model, wide-lane-only ambiguity resolution does not really pay off for the geometryfixed model.

Our analyses of the ambiguity-float and ambiguityfixed least-squares PPP-RTK estimators, also facilitated a comparison with the UPD/FCB-estimation method. Although the UPD/FCB model is as valid as the other models discussed, it was shown that the UPD/FCB estimation method cannot be accepted as a proper PPPRTK estimation method, since it does not do what it is supposed to do, namely to guarantee that the expectation of the user-ambiguity float solution is integer.
The reason lies in the fact that the used frac-operator (cf. 100) is not a proper frac-operator. Only in two special cases will this operator reduce to that of a proper frac-operator. These two cases are when only a single station is used $(n=1)$ or when the ambiguity-fixed solution $\check{\tilde{a}}_{r}^{p s}$ is used instead of the ambiguity-float solution $\hat{\tilde{a}}_{r}^{p s}$ (cf. 78).

Acknowledgements This work has benefitted from the many fruitful PPP-RTK discussions we had with our colleagues from the Curtin GNSS Research Centre. The first author is the recipient of an Australian Research Council (ARC) Federation Fellowship (project number FF0883188). This work has been done in the context of the Positioning Program Project 1.01 "New carrier phase processing strategies for achieving precise and reliable multi-satellite, multi-frequency GNSS/RNSS positioning in Australia" of the Cooperative Research Centre for Spatial Information (CRC-SI). All this support is gratefully acknowledged.

\section{Appendix}

Proof of Lemma 2 As the phase observations in (74) are reserved for the ambiguities $\tilde{a}_{r}$, the float solutions of $d \tilde{t}_{r}$ and $\tilde{\iota}_{r}$ are, respectively, determined as the IF and GF combinations of the code observations only, that is

$$
\begin{aligned}
& d \hat{\tilde{t}}_{r, G F}=-\left[\mu_{I F}^{T} \otimes D_{m}^{T}\right] p_{r}, \\
& \hat{\tilde{\iota}}_{r, G F}=+\left[\mu_{G F}^{T} \otimes D_{m}^{T}\right] p_{r}
\end{aligned}
$$

which gives the first expression of (76). The second expression (76) follows by substituting the preceding equations into

$\hat{\tilde{a}}_{r, G F}=\left[\Lambda^{-1} \otimes D_{m}^{T}\right]\left[\phi_{r}+\left[e \otimes I_{m-1}\right] d \hat{\tilde{t}}_{r, G F}+\left[\mu \otimes I_{m-1}\right] \hat{\tilde{\iota}}_{r, G F}\right],(115)$ together with the identity $e \mu_{I F}^{T}=I_{2}-\mu \mu_{G F}^{T}$.

An application of the (co)variance propagation law to (76) gives (77).

Proof of Lemma 3 Upon resolving the DD ambiguities $\check{\tilde{z}}_{r}(r=$ $1, \ldots, n)$, with $\check{\tilde{z}}_{1}=0,2(m-1)(n-1)$ redundant model's misclosures contribute to the estimation procedure, namely

$t_{\tilde{z}_{r}}=\hat{\tilde{z}}_{r}-\check{\tilde{z}}_{r}, \quad r=2, \ldots, n$

According to the least-squares conditional adjustment, the unbiased estimators $\hat{\tilde{a}}_{r, G F}$ and $d \hat{\tilde{t}}_{r, G F}$ are corrected by the above misclosures to provide the best linear unbiased estimators (BLUEs) $\check{\tilde{a}}_{r, G F}$ and $d \check{\tilde{t}}_{r, G F}$ (Teunissen, 2000). Adding the corrections, the BLUEs must remain unbiased and get uncorrelated with the underlying misclosures, see Teunissen and Khodabandeh (2013, p. 463). The unique corrections must therefore fulfill two conditions: 1) they must be zero-mean and 2) their covariances with the misclosures must be identical to those between the float estimators and the misclosures with a negative sign. Proposing the following corrections

$$
\begin{aligned}
& \epsilon_{\tilde{a}_{r}}=\check{\tilde{z}}_{r}-\hat{\tilde{a}}_{r, G F}+\frac{1}{n} \sum_{j=1}^{n}\left(\hat{\tilde{a}}_{j, G F}-\check{\tilde{z}}_{j}\right) \\
& \epsilon_{d \tilde{t}_{r}}=-Q_{d \tilde{\hat{t}}_{r}}^{G F} Q_{\tilde{\tilde{a}}_{r}} Q_{\tilde{\tilde{a}}_{r}}^{G F-1}\left(\hat{\tilde{\tilde{a}}}_{r, G F}-\tilde{\tilde{a}}_{r, G F}\right)
\end{aligned}
$$

their zero-mean property follows, respectively, from

$\mathrm{E}\left(\hat{\tilde{a}}_{j, G F}\right)=\check{\check{z}}_{j}-\tilde{\delta}, \quad j=1, \ldots, n$

$\mathrm{E}\left(\hat{\tilde{a}}_{r, G F}\right)=\mathrm{E}\left(\tilde{\tilde{a}}_{r, G F}\right)$, 
while the second-property follows, respectively, from

$Q_{\epsilon_{\tilde{a}_{r}}, t_{\tilde{z}_{r}}}^{G F}=-Q_{\tilde{\tilde{a}}_{r}, t_{\tilde{z}_{r}}}^{G F}$
$Q_{\epsilon_{d \tilde{t}_{r}}^{G F}, t_{\tilde{z}_{r}}}^{G F}=-Q_{d \tilde{t}_{r}, t_{\tilde{z}_{r}}^{G}}^{G F}$

Accordingly, the fixed solutions are obtained as

$\check{\tilde{a}}_{r, G F}=\hat{\tilde{a}}_{r, G F}+\epsilon_{\tilde{a}_{r}}$

$d \tilde{\tilde{t}}_{r}=d \hat{\tilde{t}}_{r}+\epsilon_{d \tilde{t}_{r}}$

which proves (78). Applying the (co)variance propagation law to (78) gives (79).

Proof of Table 3 We first prove the geometry-free results where again use is made of the GNSS misclosure concept (Khodabandeh and Teunissen, 2014). In the $k$-epoch case, as the ambiguities are assumed constant in time, any (co)variance matrix $Q_{\hat{x} \hat{y}}^{G F}$ is corrected according to the least-squares conditional adjustment as

$Q_{\hat{x} \hat{y}}^{G F}[k]=Q_{\hat{x} \hat{y}}^{G F}-\left[\frac{k-1}{k}\right] Q_{\hat{x}}^{G F} Q_{\tilde{a}_{r}}^{G F-1} Q_{\hat{\tilde{a}}_{r} \hat{\tilde{a}}_{r}}^{G F-1} Q_{\tilde{\hat{a}}_{r} \hat{y}}^{G F}$

Setting $\hat{y}=\hat{\tilde{a}}_{r}$, the above equation is specialized to

$Q_{\hat{x} \tilde{\tilde{a}}_{r}}^{G F}[k]=\frac{1}{k} Q_{\hat{x} \tilde{\tilde{a}}_{r}}^{G F}$

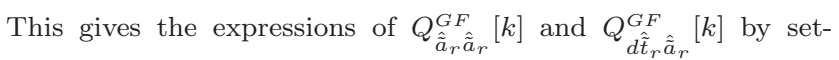
ting $\hat{x}=\hat{\tilde{a}}_{r, G F}$ and $\hat{x}=d \hat{\tilde{t}}_{r, G F}$, respectively. The expression of $Q_{d \tilde{t}_{r}}^{G F} d \hat{\tilde{t}}_{r}[k]$ follows by setting $\hat{x}=\hat{y}=d \hat{\tilde{t}}_{r}$ in (121), together with the identity

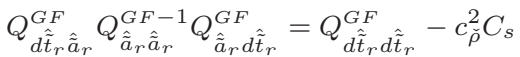

We now prove the geometry-based results. To do so, we first formulate the $[k(m-1)-\nu](n-1)$ redundant misclosures brought by the geometry-based model (cf. Table 2 ). The geometry parametrization $\Delta \tilde{x}_{r}$ gives $(m-1-\nu)(n-1)$ misclosures as

$t_{\hat{g}_{r}}=\left(D_{m}^{T} G\right)^{\perp T}\left[\frac{1}{k} \sum_{i=1}^{k}\left(d \hat{\tilde{t}}_{r, G F}(i)-d \hat{\tilde{t}}_{1, G F}(i)\right)\right], r=2, \ldots, n(12$

while the time-invariance assumption on $\Delta \tilde{x}_{r}$ gives $(k-1)(m-$ 1) $(n-1)$ misclosures as

$t_{i, r}=\left[d \hat{\tilde{t}}_{r, G F}(i)-d \hat{\tilde{t}}_{1, G F}(i)\right]-\left[d \hat{\tilde{t}}_{r, G F}(1)-d \hat{\tilde{t}}_{1, G F}(1)\right]$

for $r=2, \ldots, n$ and $i=2, \ldots, k$.

Since the two misclosure vectors $t_{\hat{g}}=\left[t_{\hat{g}_{2}}^{T}, \ldots, t_{\hat{g}_{n}}^{T}\right]^{T}$ and $t=\left[t_{2,2}^{T}, \ldots, t_{2, n}^{T}, \ldots, t_{k, n}^{T}\right]^{T}$ are uncorrelated, any (co)variance matrix $Q_{\hat{x} \hat{y}}^{G F}[k]$ is corrected to $Q_{\hat{x} \hat{y}}^{G B}[k]$ as

$$
\begin{array}{r}
Q_{\hat{x} \hat{y}}^{G B}[k]=Q_{\hat{x} \hat{y}}^{G F}[k]-Q_{\hat{x} t_{\hat{g}}}^{G F}[k] Q_{t_{\hat{y}} t_{\hat{g}}}^{-1} Q_{t_{\hat{y}} \hat{y}}^{G F}[k] \\
-Q_{\hat{x} t}^{G F}[k] Q_{t t}^{-1} Q_{t \hat{y}}^{G F}[k]
\end{array}
$$

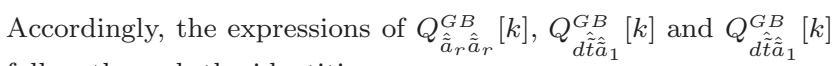
follow through the identities

$$
\begin{aligned}
& Q_{\hat{\tilde{a}}_{r} t_{\hat{g}}}^{G F}[k] Q_{t_{\hat{g}} t_{\hat{g}}}^{-1} Q_{t_{\hat{g}} \hat{\tilde{a}}_{r}}^{G F}[k]=\frac{1}{k} \frac{n-1}{n} c_{\hat{\rho}}^{2} \Lambda^{-1} e_{\mu} e_{\mu}^{T} \Lambda^{-1} \otimes \tilde{C}_{s} \\
& Q_{d \hat{t} \hat{\tilde{g}} \hat{g}}^{G F}[k] Q_{t_{\hat{g}} t_{\hat{g}}}^{-1} Q_{t_{\hat{g}} \hat{\hat{a}}_{1}}^{G F}[k]=\frac{1}{k} \frac{n-1}{n} c_{\hat{\rho}}^{2} e_{\mu}^{T} \Lambda^{-1} \otimes \tilde{C}_{s} \\
& Q_{d \tilde{t} \hat{\tilde{g}}}^{G F}[k] Q_{t_{\hat{g}} t_{\hat{g}}}^{-1} Q_{t_{\hat{g}} d \hat{\tilde{t}}}^{G F}[k]=\frac{1}{k} \frac{n-1}{n} c_{\hat{\rho}}^{2} \otimes \tilde{C}_{s}
\end{aligned}
$$

as well as

$Q_{d \tilde{\tilde{t} t}}^{G F}[k] Q_{t t}^{-1} Q_{t d \hat{\tilde{t}}}^{G F}[k]=\frac{k-1}{k} \frac{n-1}{n} c_{\check{\rho}}^{2} \otimes C_{s}$

with $Q_{\tilde{\tilde{a}}_{r} t}^{G F}[k]=0$.

Proof of Tables 4 and 5
The proof goes along the same lines as the proof of Table 3, so will not be presented here.

The exact of value of $\gamma$ The exact value of $\gamma$, introduced in Lemma 5, can be stated as

$\gamma=\frac{\left(f_{1}^{3}-f_{2}^{3}\right)^{2}}{f_{1}^{2} f_{2}^{2}\left(f_{1}+f_{2}\right)^{2}+\epsilon \eta}$

with

$\eta=\left[\frac{f_{1}+f_{2}}{f_{1}-f_{2}}\right]^{2}\left(f_{1}^{2}+f_{2}^{2}\right)\left(f_{1}^{4}+f_{2}^{4}\right)$

The approximation, given in Lemma 5 , follows by neglecting $\epsilon \eta$, compared to the first term in the denominator of (129).

\section{References}

Allison T (1991) Multi-observable processing techniques for precise relative positioning. In: Proceedings ION GPS-91. Albuquerque, New Mexico, 11-13 September, pp 715-725

Baarda W (1973) S-transformations and Criterion Matrices. Tech. rep., Netherlands Geodetic Commission, Publ. on Geodesy, New Series, Vol. 5(1), Delft

Bertiger W, Desai SD, Haines B, Harvey N, Moore AW, Owen S, Weiss JP (2010) Single receiver phase ambiguity resolution with GPS data. J Geod 84(5):327-337

Beutler G, Bock H, Dach R, Fridez P, Gade A, Hugentobler U, Jaggi A, Meindl M, Mervart L, Prange L, Schaer S, Springer T, Urschl P, Walser P (2007) Bernese GPS software version 5.0. Astron. Inst., Univ. of Bern, Bern, Switzerland

Bisnath S, Collins P (2012) Recent developments in precise point positioning. Geomatica 66(2):103-111

Bisnath S, Gao Y (2008) Current state of precise point positioning and future prospects and limitations. In: Observing our changing earth, IAG Symp 133, pp 615-623

Collins P (2008) Isolating and estimating undifferenced GPS integer ambiguities. In: Proc. ION NTM, pp 720-732

Collins P, Lahaye F, Heroux P, Bisnath S (2008) Precise point positioning with ambiguity resolution using the decoupled clock model. In: Proceedings of the 21st International Technical Meeting of the Satellite Division of The Institute of Navigation (ION GNSS 2008), pp 1315-1322

Ge M, Gendt G, Rothacher M, Shi C, Liu J (2008) Resolution of GPS carrier-phase ambiguities in precise point positioning (PPP) with daily observations. J Geod 82(7):389-399

Geng J (2011) Rapid integer ambiguity resolution in GPS precise point positioning. PhD thesis, University of Nottingham, UK

Geng J, Bock Y (2013) Triple-frequency GPS precise point positioning with rapid ambiguity resolution. J Geod 87(5):449-460

Geng J, Meng X, Dodson A, Teferle F (2010) Integer ambiguity resolution in precise point positioning: method comparison. J Geod 84(9):569-581

Geng J, Teferle FN, Meng X, Dodson AH (2011) Towards PPPRTK: Ambiguity resolution in real-time precise point positioning. Adv Space Res 47(10):1664-1673

Geng J, Shi C, Ge M, Dodson AH, Lou Y, Zhao Q, Liu J (2012) Improving the estimation of fractional-cycle biases for ambiguity resolution in precise point positioning. J Geod 86(8):579589

Hernandez-Pajares M, Juan JM, Sanz J, Colombo OL (2000) Application of ionospheric tomography to real-time GPS carrier-phase ambiguities resolution, at scales of 400-1000 $\mathrm{km}$ and with high geomagnetic activity. Geophys Res Lett 27(13):2009-2012 
Hofmann-Wellenhof B, Lichtenegger H, Wasle E (2008) GNSS: Global Navigation Satellite Systems: GPS, Glonass, Galileo, and More. Springer, New York

de Jonge PJ (1998) A processing strategy for the application of the GPS in networks. PhD thesis, Delft University of Technology, Publication on Geodesy, 46, Netherlands Geodetic Commission, Delft

Jonkman N, Teunissen P, Joosten P, Odijk D (2000) GNSS long baseline ambiguity resolution: impact of a third navigation frequency. In: Geodesy Beyond 2000, IAG Symp 121, pp 349-354

Khodabandeh A, Teunissen PJG (2014) Single-Epoch GNSS Array Integrity: an Analytical Study. IAG Symp 142, accepted for publication

Kouba J, Heroux P (2001) Precise point positioning using IGS orbit and clock products. GPS solutions 5(2):12-28

Lannes A, Prieur JL (2013) Calibration of the clock-phase biases of GNSS networks: the closure-ambiguity approach. J Geod 87(8):709-731

Lannes A, Teunissen PJG (2011) GNSS algebraic structures. J Geod 85(5):273-290

Laurichesse D (2011) The CNES real-time PPP with undifferenced integer ambiguity resolution demonstrator. In: Proceedings of the ION GNSS, pp 654-662

Laurichesse D, Mercier F (2007) Integer ambiguity resolution on undifferenced GPS phase measurements and its application to PPP. In: Proceedings of the 20th International Technical Meeting of the Satellite Division of The Institute of Navigation (ION GNSS 2007), pp 839-848

Laurichesse D, Mercier F, Berthias J, Broca P, Cerri L, CNES F (2009) Integer ambiguity resolution on undifferenced GPS phase measurements and its application to PPP and satellite precise orbit determination. Navigation 56(2):135-149

Li T, Wang J, Laurichesse D (2013a) Modeling and quality control for reliable precise point positioning integer ambiguity resolution with GNSS modernization. GPS Solut pp 1-14

Li X, Ge M, Zhang H, Wickert J (2013b) A method for improving uncalibrated phase delay estimation and ambiguity-fixing in real-time precise point positioning. J Geod 87(5):405-416

Li X, Ge M, Lu C, Zhang Y, Wang R, Wickert J, Schuh H (2014) High-rate GPS seismology using real-time precise point positioning with ambiguity resolution. Geoscience and Remote Sensing, IEEE Transactions on, pp 1-15, doi: 10.1109/TGRS.2013.2295373

Loyer S, Perosanz F, Mercier F, Capdeville H, Marty JC (2012) Zero-difference GPS ambiguity resolution at CNES-CLS IGS Analysis Center. J Geod 86(11):991-1003

Mervart L, Lukes Z, Rocken C, Iwabuchi T (2008) Precise Point Positioning with ambiguity resolution in real-time. In: Proceedings of ION GNSS, pp 397-405

Mervart L, Rocken C, Iwabuchi T, Lukes Z, Kanzaki M (2013) Precise Point Positioning with Fast Ambiguity ResolutionPrerequisites, Algorithms and Performance. In: Proceedings of ION GNSS, pp 1176-1185

Odijk D (2002) Fast precise GPS positioning in the presence of ionospheric delays. Ph.D. thesis, Delft University of Technology, Publication on Geodesy, 52, Netherlands, Geodetic Commission, Delft

Odijk D, Teunissen PJG (2008) ADOP in closed form for a hierarchy of multi-frequency single-baseline GNSS models. J Geod 82(8):473-492

Odijk D, Teunissen PJG, Zhang B (2012) Single-frequency integer ambiguity resolution enabled GPS precise point positioning. Journal of Surveying Engineering 138(4):193-202

Odijk D, Arora BS, Teunissen PJG (2014a) Predicting the success rate of long-baseline GPS+Galileo (partial) ambiguity resolution. The Journal of Navigation pp 1-17,
doi:10.1017/S037346331400006X

Odijk D, Teunissen PJG, Khodabandeh A (2014b) SingleFrequency PPP-RTK: Theory and Experimental Results. IAG Symposium Earth on the Edge: Science for a Sustainable Planet 139:571-578

Rao CR (1973) Linear statistical inference and its applications, vol 2nd ed. John Wiley \& Sons

Shi J (2012) Precise point positioning integer ambiguity resolution with decoupled clocks. PhD thesis, University of Calgary, Canada

Shi J, Gao Y (2013) A comparison of three PPP integer ambiguity resolution methods. GPS Solut (published online)

Teunissen PJG (1985) Generalized inverses, adjustment, the datum problem and S-transformations. In: Optimization and Design of Geodetic Networks, EW Grafarend and F Sanso (Eds), Springer

Teunissen PJG (1995) The least-squares ambiguity decorrelation adjustment: a method for fast GPS integer ambiguity estimation. J Geod 70(1-2):65-82

Teunissen PJG (1997a) A canonical theory for short GPS baselines. Part I: The baseline precision. J Geod 71(6):320-336

Teunissen PJG (1997b) A canonical theory for short GPS baselines. Part IV: Precision versus reliability. J Geod 71(9):513525

Teunissen PJG (1997c) The geometry-free GPS ambiguity search space with a weighted ionosphere. J Geod 71(6):370-383

Teunissen PJG (1997d) On the GPS widelane and its decorrelating property. J Geod 71(9):577-587

Teunissen PJG (1998) Success probability of integer GPS ambiguity rounding and bootstrapping. J Geod 72(10):606-612

Teunissen PJG (1999) An optimality property of the integer leastsquares estimator. J Geod 73(11):587-593

Teunissen PJG (2000) Adjustment Theory: an Introduction. Delft University Press, series on Mathematical Geodesy and Positioning

Teunissen PJG (2002) The parameter distributions of the integer GPS model. J Geod 76(1):41-48

Teunissen PJG (2012) A-PPP: array-aided precise point positioning with global navigation satellite systems. Signal Processing, IEEE Transactions on 60(6):2870-2881

Teunissen PJG, de Bakker PF (2012) Single-receiver singlechannel multi-frequency GNSS integrity: outliers, slips, and ionospheric disturbances. J Geod pp 1-17

Teunissen PJG, Khodabandeh A (2013) BLUE, BLUP and the Kalman filter: some new results. J Geod 87(5):461-473

Teunissen PJG, Odijk D, Zhang B (2010) PPP-RTK: Results of CORS Network-Based PPP with Integer Ambiguity Resolution. J Aeronaut, Astronaut Aviat 42(4):223-229

Wubbena G (1989) The GPS adjustment software packageGEONAP-concepts and models. In: International Geodetic Symposium on Satellite Positioning 1, Las Cruces, New Mexico, 13-17 March, pp 452-461

Wubbena G, Schmitz M, Bagg A (2005) PPP-RTK: Precise Point Positioning using state-space representation in RTK networks. In: Proceedings of ION GNSS, pp 13-16

Zhang B, Teunissen PJG, Odijk D (2011) A novel un-differenced PPP-RTK concept. J Navig 64(S1):S180-S191

Zhang X, Li P (2013) Assessment of correct fixing rate for precise point positioning ambiguity resolution on a global scale. J Geod 87(6):579-589

Zumberge JF, Heflin MB, Jefferson DC, Watkins MM, Webb FH (1997) Precise point positioning for the efficient and robust analysis of GPS data from large networks. J Geophys Res 102:5005-5017 